\title{
A Rhodium(I) Catalyzed Ene-Allene Carbocyclization Strategy for the Formation of Azepines and Oxepines
}

\author{
Kay M. Brummond,* Hongfeng Chen, Branko Mitasev, Anthony D. Casarez \\ Department of Chemistry, University of Pittsburgh, Pittsburgh, PA 15260
}

\section{Supporting Information}

Part 1

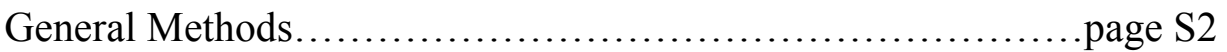

Synthesis of compounds 5, 7, 9, and 13.............page S3-S17

Synthesis of compounds $\mathbf{6 , 8}, \mathbf{1 0}$, and 16............page S17-S31

Part 2

${ }^{1} \mathrm{H}$ NMR and ${ }^{13} \mathrm{C}$ NMR spectral data for all new compounds......page S32-S63

(Spectra of the compounds are ordered according to the

compound numbers from the lowest to the highest, and do

not directly correlate with the ordering of experimental

procedures.) 


\section{General Methods}

Unless otherwise noted, all reactions were carried out under nitrogen atmosphere. All commercially available compounds were purchased from Aldrich Chemical Co., GFS Chemicals, Strem Chemicals, Acros Organics and Advanced Chemtech and used as received, unless otherwise specified. Tetrahydrofuran (THF), diethyl ether $\left(\mathrm{Et}_{2} \mathrm{O}\right)$ and dichloromethane $\left(\mathrm{CH}_{2} \mathrm{Cl}_{2}\right)$ were purified with alumina using the Sol-Tek ST-002 solvent purification system. Toluene and dichloroethane were freshly distilled from $\mathrm{CaH}_{2}$ prior to use. Purification of the compounds by flash chromatography was performed by using silica gel (32-63 $\mu \mathrm{m}$ particle size, $60 \AA$ pore size) purchased from SAI. ${ }^{1}$ TLC analyses were performed on EM Science Silica Gel $60 \mathrm{~F}_{254}$ plates $(250 \mu \mathrm{m}$ thickness $)$. HPLC purification was performed on a Varian-Prostar 210 instrument using a Varian Microsorb Dynamax 100-5 Si column (5 $\mu \mathrm{m}$ packing, $250 \mathrm{~mm}$ x $10 \mathrm{~mm}$ ). Gas chromatography was performed on a Shimadzu GC17A gas chromatograph with a $15 \mathrm{ft}$ RTX-5 column that contains 5\% diphenyl crossbonds and 95\% dimethylpolysiloxane stationary phase with an internal diameter of $0.25 \mathrm{~mm}$ and film thickness of $0.25 \mu \mathrm{m}$. All ${ }^{1} \mathrm{H}$ and ${ }^{13} \mathrm{C}$ spectra were obtained on Bruker Avance $300 \mathrm{MHz}$, and chemical shifts $(\delta)$ reported relative to residual solvent peak $\mathrm{CHCl}_{3}, \mathrm{DMSO}, \mathrm{CH}_{2} \mathrm{Cl}_{2}$ or $\mathrm{C}_{6} \mathrm{H}_{6}$. All NMR spectra were obtained at room temperature unless otherwise specified. IR spectra were obtained using a Nicolet Avatar E.S.P. 360 FT-IR. EI mass spectrometry was performed on a Micromass Autospec high resolution mass spectrometer. The glassware was typically oven-dried or flame-dried.

\footnotetext{
${ }^{1}$ Still, W. C.; Kahn, M.; Mitra, A. J. Org. Chem. 1978, 43, 2923.
} 
In some cases (compound 7, entries 11, 12 and 13, compound 8, entry 12) mixtures of diastereomers were obtained and were not separated for characterization purposes. Consequently, the spectroscopic data has been reported as a mixture of diastereomers. Where possible, resonances attributed to a single diastereomer are labeled accordingly: * denotes one diastereomer and ** denotes a second diastereomer. Where possible, the diastereomeric ratios were measured by integration of the resonances in the ${ }^{1} \mathrm{H}$ NMR. 


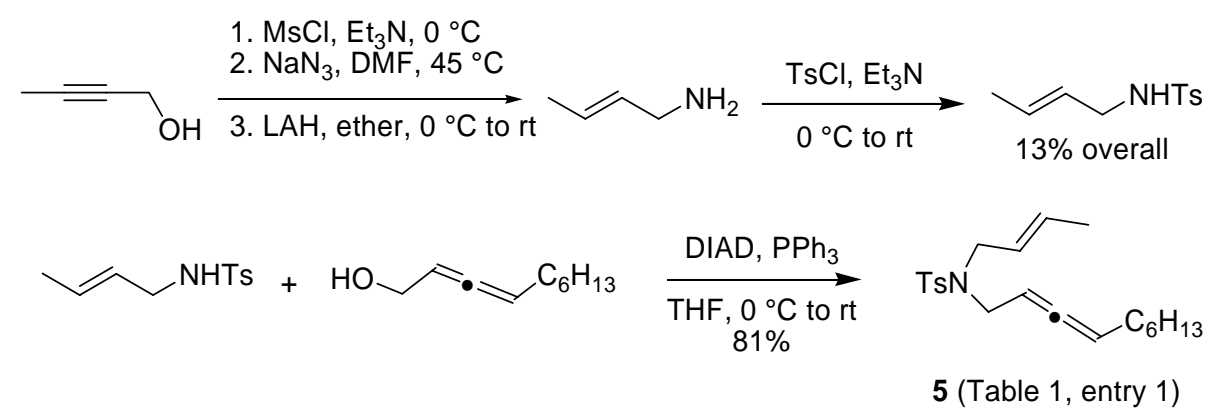

$N$-[(E)-2-Butenyl]- $N$-2,3-decadienyl-4-methylbenzenesulfonamide (5, Table 1, entry 1): Methanesulfonyl chloride $(9.3 \mathrm{~mL}, 120 \mathrm{mmol})$ was added dropwise over 10 min via a syringe into a cold $\left(0{ }^{\circ} \mathrm{C}\right)$ solution of 2-butyn-1-ol $(7.00 \mathrm{~g}, 100 \mathrm{mmol})$ and triethylamine $(20.0 \mathrm{~mL}, 144 \mathrm{mmol})$ in methylene chloride $(200 \mathrm{~mL})$. The mixture was stirred at $0{ }^{\circ} \mathrm{C}$ for $1 \mathrm{~h}$ until the TLC indicated total consumption of the starting materials, and was quenched with sat. $\mathrm{NH}_{4} \mathrm{Cl}$ solution $(20 \mathrm{~mL})$. The mixture was warmed to rt, washed successively with a mixture of brine : $\mathrm{NaHCO}_{3}$ : water $(1: 1: 1,2 \times 30 \mathrm{~mL})$, sat. $\mathrm{NaHCO}_{3}(2 \times 30 \mathrm{~mL})$, and brine $(2 \times 30 \mathrm{~mL})$, dried $\left(\mathrm{MgSO}_{4}\right)$, filtered and concentrated in vacuo to afford crude 2-butynyl methanesulfonate as a yellow oil. This oil was then dissolved in dry DMF $(100 \mathrm{~mL})$ and cooled to $0{ }^{\circ} \mathrm{C}$. Sodium azide $(20.0 \mathrm{~g}, 308 \mathrm{mmol})$ was added and the mixture was stirred at $\mathrm{rt}$ for $12 \mathrm{~h}$ then at $45^{\circ} \mathrm{C}$ for $7 \mathrm{~h}$. The mixture was cooled to rt, diluted with water $(200 \mathrm{~mL})$, and extracted with ether $(5 \times 100 \mathrm{~mL})$. The combined ether layers was washed with water $(2 \times 50 \mathrm{~mL})$, brine $(3 \times 50 \mathrm{~mL})$, dried $\left(\mathrm{MgSO}_{4}\right)$, filtered and concentrated in vacuo to afford crude 1-azido-2-butyne as a yellow oil. This oil was dissolved in anhydrous ether $(200 \mathrm{~mL})$ and cooled to $0{ }^{\circ} \mathrm{C}$. LAH $(7.60$ g, $200 \mathrm{mmol}$ ) was added cautiously in small portions (Caution! Rapid gas evolution occurs!). Following the addition of LAH the mixture was allowed to warm to $\mathrm{rt}$ and stirred for $6 \mathrm{~h}$. The reaction was then cooled to $0{ }^{\circ} \mathrm{C}$ and quenched with a minimum amount of water to the point where the precipitate turned from grey to white. The solid 
was then removed by filtration, and rinsed with ether. The combined filtrate was dried $\left(\mathrm{Na}_{2} \mathrm{SO}_{4}\right)$ and filtered. Simple distillation of the solvent afforded crude $(E)$-2butenylamine as a yellow oil. This oil was dissolved in methylene chloride $(200 \mathrm{~mL})$ with triethyl amine $(15 \mathrm{~mL}, 108 \mathrm{mmol})$. The mixture was cooled to $0{ }^{\circ} \mathrm{C}$ then 4 methylbenzenesulfonyl chloride $(3.76 \mathrm{~g}, 19.7 \mathrm{mmol})$ was added in one portion. The mixture was stirred at $\mathrm{rt}$ for overnight and concentrated in vacuo to a yellow thick oil. The residue was dissolved in ethyl acetate $(200 \mathrm{~mL})$, washed with water $(2 \times 30 \mathrm{~mL})$, sat. $\mathrm{NH}_{4} \mathrm{Cl}(2 \times 30 \mathrm{~mL})$, brine $(2 \times 30 \mathrm{~mL})$, dried $\left(\mathrm{Na}_{2} \mathrm{SO}_{4}\right)$, filtered and concentrated. Flash chromatography $\left(\mathrm{SiO}_{2}\right.$, hexanes : ether $\left.=1: 1\right)$ afforded $N$-[(E)-2-butenyl]-4methylbenzenesulfonamide as a colorless solid (2.80 g, 13\% overall yield for 4 steps).

\section{General procedure for the preparation of ene-allenes via a Mitsunobu reaction: ${ }^{2}$}

To a solution of $N$-[(E)-2-butenyl]-4-methylbenzenesulfonamide (1.73 g, 7.70 mmol, 1.00 eq.), $\mathrm{PPh}_{3}$ (2.73 g, 10.4 mmol, 1.35 eq.) and 2,3-decadien-1-ol (1.36 g, 8.80 mmol, 1.15 eq. $)$ in $\mathrm{THF}(25 \mathrm{~mL})$ at $0 \quad{ }^{\circ} \mathrm{C}$ was added dropwise diisopropylazodicarboxylate (DIAD, $2.04 \mathrm{~mL}, 10.4 \mathrm{mmol}, 1.35$ eq.). The resulting solution was allowed to slowly warm to rt overnight. THF was removed in vacuo and the residue was purified by flash chromatography $\left(\mathrm{SiO}_{2}\right.$, hexanes : ethyl acetate $\left.=90: 10\right)$ to afford title compound 5 (Table 1, entry 1 ) as a slightly yellow oil $(2.25 \mathrm{~g}, 81 \%)$ : ${ }^{1} \mathrm{H}$ NMR (300 MHz, $\left.\mathrm{CDCl}_{3}\right) \delta 7.70(\mathrm{~d}, J=8.3 \mathrm{~Hz}, 2 \mathrm{H}), 7.28(\mathrm{~d}, J=8.3 \mathrm{~Hz}, 2 \mathrm{H}), 5.57$ (dtq, $J$ $=1.2,6.5,15.2 \mathrm{~Hz}, 1 \mathrm{H}), 5.29(\mathrm{dtq}, J=1.6,5.0,15.2 \mathrm{~Hz}, 1 \mathrm{H}), 5.08(\mathrm{dtt}, J=2.3,6.4,6.8$ $\mathrm{Hz}, 1 \mathrm{H}), 4.84$ (dtt, $J=2.8,6.3,6.8 \mathrm{~Hz}, 1 \mathrm{H}), 3.81-3.77(\mathrm{~m}, 4 \mathrm{H}), 2.42(\mathrm{~s}, 3 \mathrm{H}), 1.95$ (dq, $J=$ 2.7, $7.4 \mathrm{~Hz}, 2 \mathrm{H}), 1.64(\mathrm{dd}, J=1.5,6.5 \mathrm{~Hz}, 3 \mathrm{H}), 1.35-1.22(\mathrm{~m}, 8 \mathrm{H}), 0.89(\mathrm{t}, J=7.0 \mathrm{~Hz}$,

\footnotetext{
${ }^{2}$ Pagenkopf, B.L.; Belanger, D.B.; O'Mahony, D.J.R.; Livinghouse, T. Synthesis, 2000, 1009.
} 
$3 \mathrm{H}) ;{ }^{13} \mathrm{C}$ NMR $\left(75 \mathrm{MHz}, \mathrm{CDCl}_{3}\right) \delta 205.2,142.9,137.9,130.3,129.5,127.2,125.4,92.4$, $86.4,48.3,46.0,31.6,29.1,28.7,28.5,22.5,21.4,17.5,13.9$; IR (neat) $v$ 2927, 1962, 1348, $1161 \mathrm{~cm}^{-1}$; EI-HRMS calcd for $\mathrm{C}_{21} \mathrm{H}_{31} \mathrm{NO}_{2} \mathrm{~S}\left[\mathrm{M}^{+}\right] \mathrm{m} / \mathrm{z}$ 361.2092, found 361.2076.
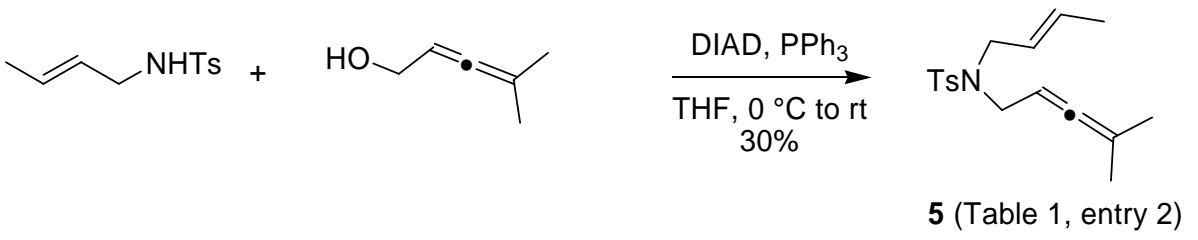

$N$-[(E)-2-Butenyl]- $N$-2,3-pentadienyl-4-methylbenzenesulfonamide $\quad$ (5, Table

1, entry 2): This compound was prepared following the general procedure for the preparation of ene-allenes via a Mitsunobu reaction (page S5) from $N$-[(E)-2-butenyl]-4methylbenzenesulfonamide $\left(0.250 \mathrm{~g}, 1.10 \mathrm{mmol}, 1.00\right.$ eq.), $\mathrm{PPh}_{3}(0.722 \mathrm{~g}, 2.75 \mathrm{mmol}$, 2.50 eq.), 4-methyl-2,3-pentadien-1-ol (0.220 g, $2.20 \mathrm{mmol})$ and DIAD (0.54 mL, 2.8 mmol, 2.5 eq.) as a colorless oil $(0.100 \mathrm{~g}, 30 \%)$ : ${ }^{1} \mathrm{H}$ NMR $\left(300 \mathrm{MHz}, \mathrm{CDCl}_{3}\right) \delta 7.69$ (d, $J=8.2 \mathrm{~Hz}, 2 \mathrm{H}), 7.28(\mathrm{~d}, J=8.2 \mathrm{~Hz}, 2 \mathrm{H}), 5.57(\mathrm{dq}, J=6.4,15.1 \mathrm{~Hz}, 1 \mathrm{H}), 5.32-5.25(\mathrm{~m}$, $1 \mathrm{H}), 4.72-4.67(\mathrm{~m}, 1 \mathrm{H}), 3.88-3.71(\mathrm{~m}, 4 \mathrm{H}), 2.42(\mathrm{~s}, 3 \mathrm{H}), 1.68-1.58(\mathrm{~m}, 9 \mathrm{H}) ;{ }^{13} \mathrm{C} \mathrm{NMR}$ $\left(75 \mathrm{MHz}, \mathrm{CDCl}_{3}\right) \delta 203.1,142.9,137.8,130.3,129.5,127.1,125.3,96.5,84.3,48.2$, 46.1, 21.5, 20.3, 17.7; IR (neat) $v 2917,1969,1344,1160 \mathrm{~cm}^{-1}$; EI-HRMS calcd for $\mathrm{C}_{17} \mathrm{H}_{23} \mathrm{NO}_{2} \mathrm{~S}\left[\mathrm{M}^{+}\right] \mathrm{m} / \mathrm{z} 305.1450$, found 305.1455 .
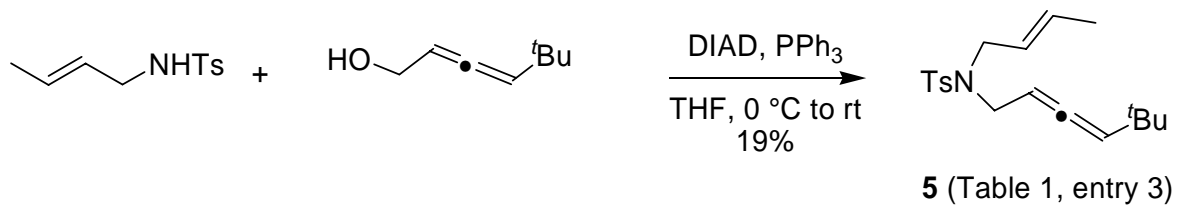

\section{$N$-[(E)-2-Butenyl]- $N$-(5,5-dimethyl-2,3-hexadienyl)-4-methylbenzenesulfo-}

namide (5, Table 1, entry 3): This compound was prepared following the general procedure for the preparation of ene-allenes via a Mitsunobu reaction (page S5) from $\mathrm{N}$ - 
[(E)-2-butenyl]-4-methylbenzenesulfonamide ( $0.235 \mathrm{~g}, 1.04$ mmol, 1.00 eq.), $\mathrm{PPh}_{3}(0.410$ g, $1.56 \mathrm{mmol}, 1.50$ eq.), 5,5-dimethyl-2,3-hexadien-1-ol (0.150 g, 1.09 mmol, 1.05 eq.) and DIAD (0.31 mL, 1.5 eq.) as a colorless oil (0.066 g, 19\%): ${ }^{1} \mathrm{H}$ NMR (300 MHz, $\left.\mathrm{CDCl}_{3}\right) \delta 7.69(\mathrm{~d}, J=8.3 \mathrm{~Hz}, 2 \mathrm{H}), 7.28(\mathrm{~d}, J=8.3 \mathrm{~Hz}, 2 \mathrm{H}), 5.57(\mathrm{dq}, J=6.5,15.2 \mathrm{~Hz}$, $1 \mathrm{H}), 5.27(\mathrm{dtt}, J=1.6,6.7,15.2 \mathrm{~Hz}, 1 \mathrm{H}), 5.11(\mathrm{dt}, J=2.3,6.2 \mathrm{~Hz}, 1 \mathrm{H}), 4.94(\mathrm{dt}, J=6.8$, $6.8 \mathrm{~Hz}, 1 \mathrm{H}), 3.92-3.72(\mathrm{~m}, 4 \mathrm{H}), 2.42(\mathrm{~s}, 3 \mathrm{H}), 1.63(\mathrm{dd}, J=1.2,6.4 \mathrm{~Hz}, 2 \mathrm{H}), 1.00(\mathrm{~s}, 9 \mathrm{H})$; ${ }^{13} \mathrm{C}$ NMR $\left(75 \mathrm{MHz}, \mathrm{CDCl}_{3}\right) \delta 202.4,142.9,137.9,130.3,129.5,127.2,125.3,104.4$, $88.5,48.5,46.2,31.7,30.3,21.4,17.5$; IR (neat) $\vee 2961,1960,1347,1160 \mathrm{~cm}^{-1}$; EIHRMS calcd for $\mathrm{C}_{19} \mathrm{H}_{27} \mathrm{NO}_{2} \mathrm{~S}\left[\mathrm{M}^{+}\right] \mathrm{m} / \mathrm{z} 333.1763$, found 333.1766 .

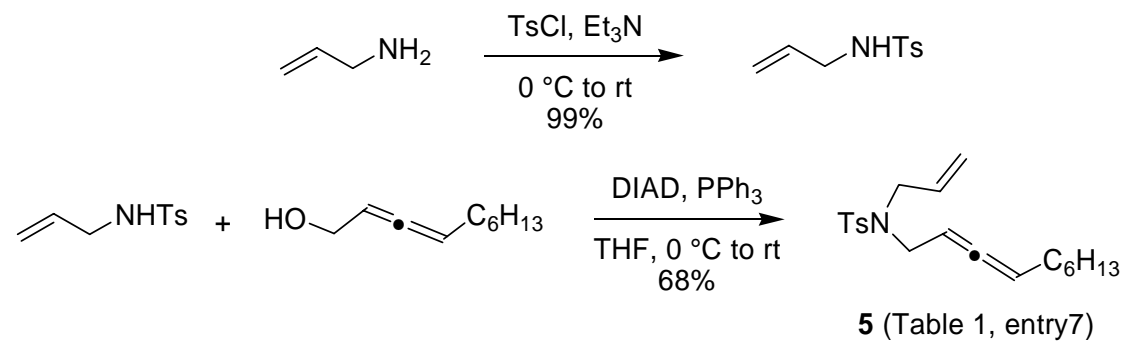

$N$-Allyl- $N$-2,3-decadienyl-4-methylbenzenesulfonamide (5, Table 1 , entry 7 ):

To a cold $\left(0{ }^{\circ} \mathrm{C}\right)$ solution of 4-methylbenzenesulfonyl chloride $(4.05 \mathrm{~g}, 21.0 \mathrm{mmol})$ in methylene chloride $(100 \mathrm{~mL})$ was added allylamine $(8.0 \mathrm{~mL}, 106 \mathrm{mmol}, 5.0$ eq. $)$. The mixture was warmed to rt and stirred for $1 \mathrm{~h}$. The solvent was removed in vacuo and the residue dissolved in ethyl acetate $(200 \mathrm{~mL})$, washed with water $(20 \mathrm{~mL})$, brine $(3 \times 20$ $\mathrm{mL})$, dried $\left(\mathrm{Na}_{2} \mathrm{SO}_{4}\right)$, filtered and concentrated in vacuo to afford $\mathrm{N}$-allyl-4methylbenzenesulfonamide as a colorless oil which solidified upon standing (4.43 g, $99 \%)$.

The title compound was prepared following the general procedure for the preparation of ene-allenes via a Mitsunobu reaction (page S5) from $N$-allyl-4- 
methylbenzenesulfonamide $(0.504 \mathrm{~g}, 2.39 \mathrm{mmol}, 1.00$ eq. $), \mathrm{PPh}_{3}(0.845 \mathrm{~g}, 3.22 \mathrm{mmol}$, 1.35 eq.), 2,3-decadien-1-ol (0.478 g, 3.10 mmol, 1.30 eq.) and DIAD (0.63 mL, 3.2 mmol, 1.4 eq.) as a light yellow oil $(0.56 \mathrm{~g}, 68 \%):{ }^{1} \mathrm{H}$ NMR $\left(300 \mathrm{MHz}, \mathrm{CDCl}_{3}\right) \delta 7.71$ (d, $J=8.3 \mathrm{~Hz}, 2 \mathrm{H}), 7.29(\mathrm{~d}, J=8.3 \mathrm{~Hz}, 2 \mathrm{H}), 5.64(\mathrm{ddt}, J=6.3,10.4,16.8 \mathrm{~Hz}, 1 \mathrm{H}), 5.20-$ $5.06(\mathrm{~m}, 3 \mathrm{H}), 4.89-4.81(\mathrm{~m}, 1 \mathrm{H}), 3.90-3.81(\mathrm{~m}, 4 \mathrm{H}), 2.43(\mathrm{~s}, 3 \mathrm{H}), 1.97-1.92(\mathrm{~m}, 2 \mathrm{H})$, 1.49-1.25 (m, 8H), $0.88(\mathrm{t}, J=7.1 \mathrm{~Hz}, 3 \mathrm{H}) ;{ }^{13} \mathrm{C}$ NMR $\left(75 \mathrm{MHz}, \mathrm{CDCl}_{3}\right) \delta 205.3,143.1$, $137.6,132.7,129.6,127.2,118.8,92.5,86.1,49.0,46.3,31.6,29.1,28.7,28.5,22.6,21.5$, 14.1; IR (neat) $\vee 3082,2927,1963,1348,1160 \mathrm{~cm}^{-1}$; EI-HRMS calcd for $\mathrm{C}_{20} \mathrm{H}_{29} \mathrm{NO}_{2} \mathrm{~S}$ $\left[\mathrm{M}^{+}\right] \mathrm{m} / \mathrm{z}$ 347.1919, found 347.1920.
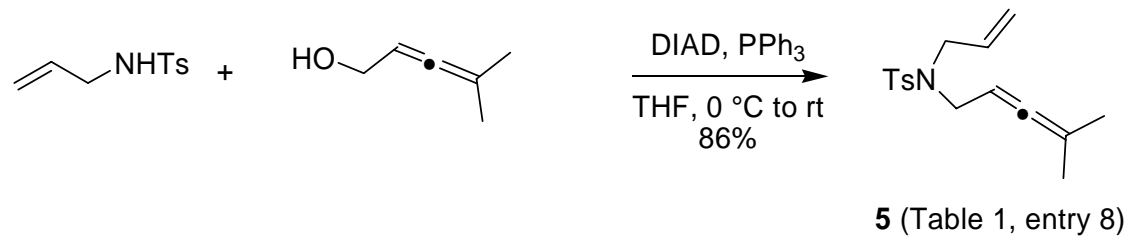

$N$-Allyl-4-methyl- $N$-(4-methyl-2,3-pentadienyl)benzenesulfonamide (5, Table 1, entry 8): This compound was prepared following the general procedure for the preparation of ene-allenes via a Mitsunobu reaction (page S5) from $N$-allyl-4methylbenzenesulfonamide $(0.501 \mathrm{~g}, 2.37 \mathrm{mmol}, 1.00$ eq. $), \mathrm{PPh}_{3}(0.839 \mathrm{~g}, 3.20 \mathrm{mmol}$, 1.35 eq.), 4-methyl-2,3-pentadien-1-ol (0.303 g, $3.08 \mathrm{mmol}, 1.30$ eq.) and DIAD (0.63 $\mathrm{mL}, 3.2 \mathrm{mmol}, 1.4$ eq.) as a light yellow oil $(0.60 \mathrm{~g}, 86 \%)$ : ${ }^{1} \mathrm{H} \mathrm{NMR}\left(300 \mathrm{MHz}, \mathrm{CDCl}_{3}\right)$ $\delta$ 7.74-7.69 (m, 2H), $7.29(\mathrm{~d}, J=8.0 \mathrm{~Hz}, 2 \mathrm{H}), 5.67(\mathrm{ddt}, J=6.1,10.3,17.0 \mathrm{~Hz}, 1 \mathrm{H}), 5.18$ (ddt, $J=1.3,2.7,9.4 \mathrm{~Hz}, 1 \mathrm{H}), 5.14-5.11(\mathrm{~m}, 1 \mathrm{H}), 4.79-4.71(\mathrm{~m}, 1 \mathrm{H}), 3.86(\mathrm{~d}, J=63 \mathrm{~Hz}$, 2H), $3.78(\mathrm{~d}, J=6.9 \mathrm{~Hz}, 2 \mathrm{H}), 2.43(\mathrm{~s}, 3 \mathrm{H}), 1.65(\mathrm{~d}, J=2.8 \mathrm{~Hz}, 6 \mathrm{H}) ;{ }^{13} \mathrm{C} \mathrm{NMR}(75 \mathrm{MHz}$, $\left.\mathrm{CDCl}_{3}\right) \delta 203.4,143.0,137.9,133.0,129.6,127.2,118.6,96.6,84.4,49.0,46.6,21.4$, 
20.2; IR (neat) $v 3082,2913,1968,1345,1160 \mathrm{~cm}^{-1}$; EI-HRMS calcd for $\mathrm{C}_{16} \mathrm{H}_{21} \mathrm{NO}_{2} \mathrm{~S}$ $\left[\mathrm{M}^{+}\right] \mathrm{m} / \mathrm{z}$ 291.1293, found 291.1294.

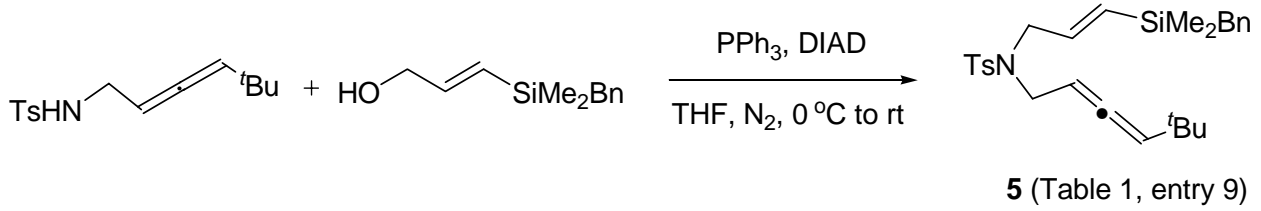

\section{$N$-[3-benzyldimethylsilyl-2-(E)-propenyl]- $N$-(5,5-Dimethyl-2,3-hexadienyl)-4-}

methyl-benzenesulfonamide (5, Table 1 , entry 9). The general procedure for the Mitsunobu reaction (page S5) was followed using $\mathrm{PPh}_{3}(240 \mathrm{mg}, 0.916 \mathrm{mmol}), \mathrm{N}-(5,5-$ Dimethyl-2,3-hexadienyl)-4-methylbenzenesulfonamide (190 mg, $0.680 \mathrm{mmol}), \quad 3-$ benzyldimethylsilyl-2-(E)-propen-1-ol (181 mg, $0.878 \mathrm{mmol})$, and DIAD (180 $\mu \mathrm{L}, 0.914$ mmol). The resulting residue was purified by silica gel chromatography (ethyl acetatehexanes 1:19-1:7) to afford $82 \mathrm{mg}$ of the title compound as a colorless oil in $79 \%$ yield. ${ }^{1} \mathrm{H} \mathrm{NMR}\left(300 \mathrm{MHz}, \mathrm{CDCl}_{3}\right) \delta: 7.70(1 / 2 \mathrm{AB} \mathrm{q}, J=8.3 \mathrm{~Hz}, 2 \mathrm{H}), 7.27(1 / 2 \mathrm{AB}$ q, $J=8.0$ $\mathrm{Hz}, 2 \mathrm{H}), 7.23-7.18(\mathrm{~m}, 2 \mathrm{H}), 7.10-7.05(\mathrm{~m}, 1 \mathrm{H}), 6.98-6.95(\mathrm{~m}, 2 \mathrm{H})$, 5.74-5.73 (m, 2H), $5.14(\mathrm{dt}, J=6.2,2.3 \mathrm{~Hz}, 1 \mathrm{H}), 4.95(\mathrm{q}, J=6.5 \mathrm{~Hz}, 1 \mathrm{H}), 3.90(\mathrm{~d}, J=2.3 \mathrm{~Hz}, 2 \mathrm{H}) 3.81$ (ddd, $J=15.1,7.7,2.2 \mathrm{~Hz}, 2 \mathrm{H}), 2.41(\mathrm{~s}, 3 \mathrm{H}), 2.08(\mathrm{~s}, 2 \mathrm{H}), 1.02(\mathrm{~s}, 9 \mathrm{H}),-0.0042(\mathrm{~s}, 3 \mathrm{H}),-$ $0.0068(\mathrm{~s}, 3 \mathrm{H}) ;{ }^{13} \mathrm{C}$ NMR (75 MHz, $\left.\mathrm{CDCl}_{3}\right)$ \%: 202.9, 143.4, 141.5, 139.9, 138.3, 132.8, $130.0,128.50,128.47,127.5,124.4,104.8,88.7,51.4,47.0,32.1,30.4,26.1,21.8,-3.1$; IR (neat) 3017, 2955, 2930, 2904, 2863, 1962, 1619, 1598, 1491, 1455, $1347 \mathrm{~cm}^{-1}$; MS m/z (\%) 468 (15), 467(46), 452 (39), 376 (33), 372 (49), 59 (100); EI-HRMS calcd for $\mathrm{C}_{27} \mathrm{H}_{37} \mathrm{NO}_{2} \mathrm{SiS}: 467.2318$; found: 467.2314 .

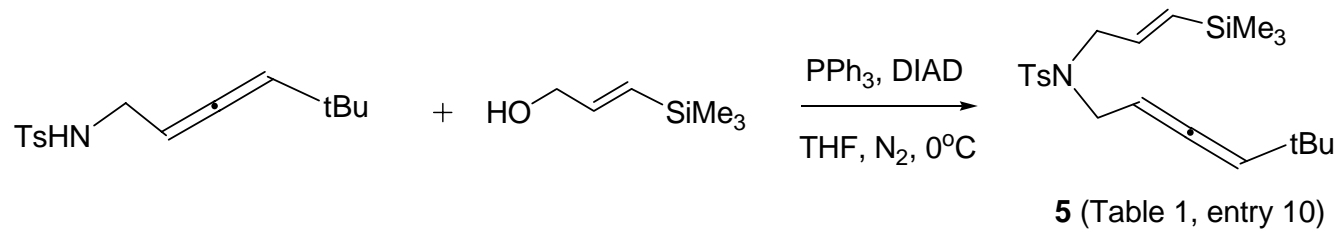




\section{$N$-(5,5-Dimethyl-2,3-hexadienyl)- $N$-[3-trimethylsilyl-2-(E)-propenyl]-4-}

methylbenzenesulfonamide ( 5 , Table 1 , entry 10$)$. The general procedure for the Mitsunobu reaction (page S5) was followed using $\mathrm{PPh}_{3}(123 \mathrm{mg}, 0.470 \mathrm{mmol}), \mathrm{N}-(5,5-$ Dimethyl-2,3-hexadienyl)-4-methylbenzenesulfonamide $\quad(98 \mathrm{mg}, \quad 0.35 \mathrm{mmol}), \quad 3-$ trimethylsilyl-2-(E)-propen-1-ol (70 $\mu \mathrm{L}, 0.46 \mathrm{mmol})$, and DIAD (93 $\mu \mathrm{L}, 0.47 \mathrm{mmol})$. The resulting residue was purified by silica gel chromatography (ethyl acetate-hexanes 1:19-1:9) to afford $91 \mathrm{mg}$ of the title compound in $67 \%$ yield. ${ }^{1} \mathrm{H}$ NMR $(300 \mathrm{MHz}$, $\left.\mathrm{CDCl}_{3}\right) \delta: 7.71(1 / 2 \mathrm{AB} \mathrm{q}, J=8.2 \mathrm{~Hz}, 2 \mathrm{H}), 7.30(1 / 2 \mathrm{AB} \mathrm{q}, J=8.2 \mathrm{~Hz}, 2 \mathrm{H}), 5.73-5.79$ (m, 2H), $5.13(\mathrm{dt}, J=6.2,2.2 \mathrm{~Hz}, 1 \mathrm{H}), 4.93(\mathrm{q}, J=6.6 \mathrm{~Hz}, 1 \mathrm{H}), 3.93-3.88(\mathrm{~m}, 2 \mathrm{H}), 3.83$ (ddd, $J=14.9,7.0,2.0 \mathrm{~Hz}, 2 \mathrm{H}), 2.43(\mathrm{~s}, 3 \mathrm{H}), 1.01(\mathrm{~s}, 9 \mathrm{H}), 0.01(\mathrm{~s}, 9 \mathrm{H}) ;{ }^{13} \mathrm{C}$ NMR $(75$ $\left.\mathrm{MHz}, \mathrm{CDCl}_{3}\right) \delta: 202.9,143.4,140.1,138.2,135.0,129.9,127.5,104.8,88.6,51.4,47.0$, 32.1, 30.4, 21.8, -1.2; IR (neat) 2956, 2863, 1957, 1440, $1350 \mathrm{~cm}^{-1}$; MS m/z (\%) 391 (10), 376 (78), 296 (100); EI-HRMS calcd for $\mathrm{C}_{21} \mathrm{H}_{33} \mathrm{NO}_{2} \mathrm{SiS}$ : 391.2001; found: 391.1998.

General procedure for $\mathrm{N}$-alkylation of $\mathrm{N}$-protected allenic amino acid methyl esters.

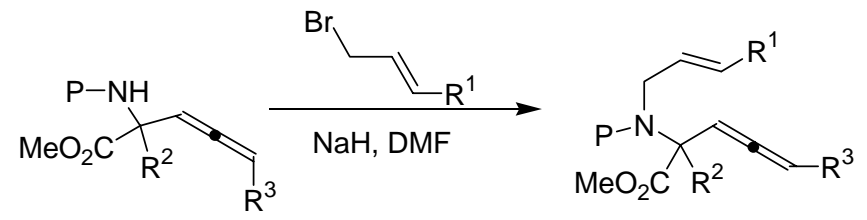

To a solution of the N-protected amino $\operatorname{acid}^{3}(1.0 \mathrm{mmol})$ in DMF $(4 \mathrm{~mL})$, was added $\mathrm{NaH}$ (2.0 mmol of a $60 \%$ dispersion in mineral oil) at $0{ }^{\circ} \mathrm{C}$ under $\mathrm{N}_{2}$ atmosphere. After $2 \mathrm{~min}$ the alkyl bromide $(1.5-2.0 \mathrm{mmol})$ was added dropwise. The reaction mixture was stirred at $\mathrm{rt}$ for $15-45 \mathrm{~min}$ and upon completion as observed by TLC was quenched by cautiously pouring into water $(50 \mathrm{~mL})$. The mixture was extracted with EtOAc $(3 \times 50$ $\mathrm{mL}$ ). The organic layers were combined, washed with brine, dried with $\mathrm{MgSO}_{4}$, and

\footnotetext{
${ }^{3}$ Brummond, K. M.; Mitasev, B. Manuscript submitted for publication.
} 
concentrated under vacuum. Purification by flash chromatography (hexanes-EtOAc, $9: 1$, $\mathrm{v} / \mathrm{v})$ afforded the desired alkylated products.

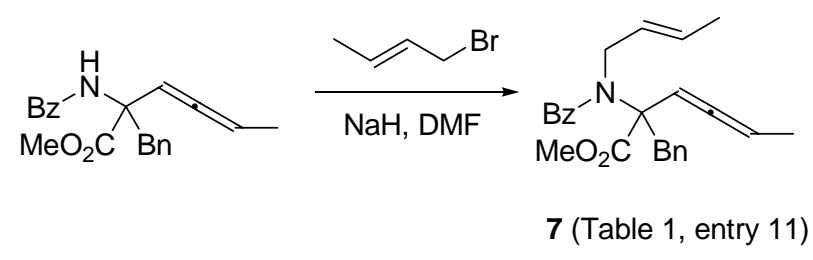

2-(Benzoyl-but-2-enylamino)-2-benzylhexa-3,4-dienoic acid methyl ester (7, Table 1, entry 11). Followed general procedure for N-alkylation of N-protected allenic amino acid methyl esters (page S10):

2-Benzoylamino-2-benzyl-hexa-3,4-dienoic acid methyl ester (92 mg, $0.27 \mathrm{mmol}), \mathrm{NaH}$ ( $60 \%$ dispersion in mineral oil, $22 \mathrm{mg}, 0.55 \mathrm{mmol}$ ), crotyl bromide ( $57 \mu \mathrm{L}, 0.55 \mathrm{mmol}$ ).

Purification by flash chromatography (hexanes-EtOAc, $9: 1$, v/v) afforded the title compound (60 mg, $56 \%)$ in diastereomeric ratio of $1.6: 1$.

${ }^{1} \mathrm{H}$ NMR (300 MHz, $\left.\mathrm{CDCl}_{3}\right)$ 8: 7.39-7.27 (m, 10H), 5.62-5.56 (m, 1H), 5.55-5.21 (m, 2H), 4.96-4.88 (m, 1H), $4.00(\mathrm{~d}, J=13.5 \mathrm{~Hz}, 1 \mathrm{H})^{*}, 3.97(\mathrm{~d}, J=13.5 \mathrm{~Hz}, 1 \mathrm{H})^{* *}, 3.77$ (s, $3 \mathrm{H}), 3.61-3.53(\mathrm{~m}, 1 \mathrm{H}), 3.44-3.36(\mathrm{~m}, 1 \mathrm{H}), 3.35(\mathrm{~d}, J=13.5 \mathrm{~Hz}, 1 \mathrm{H}), 1.80(\mathrm{dd}, J=7.1$, $3.3 \mathrm{~Hz}, 1 \mathrm{H})^{*}, 1.74(\mathrm{dd}, J=7.1,3.2 \mathrm{~Hz})^{* *}, 1.53(\mathrm{~m}, 3 \mathrm{H}) ;{ }^{13} \mathrm{C} \mathrm{NMR}\left(\mathrm{CDCl}_{3}, 75 \mathrm{MHz}\right) \delta$ : 204.7, 173.0, 172.1, 137.3, 137.0, 131.5, 131.5, 129.7, 129.0, 128.6, 128.5, 128.3, 127.7, $127.2,127.1,127.0,126.8,91.6,91.5,90.7,90.4,69.2,52.6,52.5,50.4,50.3,39.5,39.4$, 18.0, 14.2, 13.9; IR (thin film) v 2947, 1968, 1744, 1637, 1444, 1396, $1224 \mathrm{~cm}^{-1} ; \mathrm{MS} \mathrm{m} / \mathrm{z}$ (\%) 388 (6), 358 (5), 330 (33), 298 (84), 244 (60), 105 (100); EI-HRMS calcd for [M-1 ${ }^{+}$], $m / z \mathrm{C}_{25} \mathrm{H}_{26} \mathrm{NO}_{3} 388.1913$, found 388.1912 .

* denotes the major diastereomer 
** denotes the minor diastereomer

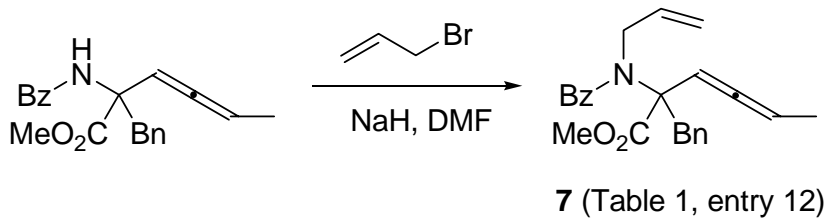

2-(Allylbenzoylamino)-2-benzylhexa-3,4-dienoic acid methyl ester (7, Table 1, entry 12). Followed general procedure for N-alkylation of N-protected allenic amino acid methyl esters (page s10):

2-Benzoylamino-2-benzyl-hexa-3,4-dienoic acid methyl ester (100mg, $0.297 \mathrm{mmol}$ ), $\mathrm{NaH}(60 \%$ dispersion in mineral oil, $24 \mathrm{mg}, 0.59 \mathrm{mmol})$, allyl bromide (40 $\mu \mathrm{L}, 0.45$ mmol) in diastereomeric ratio of $1.6: 1$.

Purification by flash chromatography (hexanes-EtOAc, $9: 1, \mathrm{v} / \mathrm{v}$ ) afforded the title compound (80 mg, 71\%).

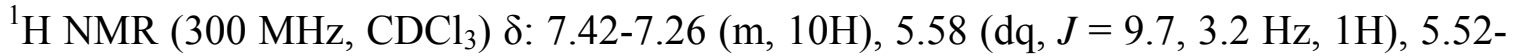
$5.41(\mathrm{~m}, 2 \mathrm{H}), 5.25-5.14(\mathrm{~m}, 1 \mathrm{H}), 5.06-5.03(\mathrm{~m}, 1 \mathrm{H}), 3.99(\mathrm{~d}, J=13.5 \mathrm{~Hz}, 1 \mathrm{H})^{*}, 3.95(\mathrm{~d}, J$ $=13.5 \mathrm{~Hz}, 1 \mathrm{H})^{* *}, 3.78(\mathrm{~s}, 3 \mathrm{H})^{* *}, 3.77(\mathrm{~s}, 3 \mathrm{H})^{*}, 3.65-3.57(\mathrm{~m}, 1 \mathrm{H}), 3.39(\mathrm{~d}, J=13.5 \mathrm{~Hz}$, 1H), 3.41-3.28 (m, 1H), $1.80(\mathrm{dd}, J=7.1,3.3 \mathrm{~Hz}, 3 \mathrm{H})^{*}, 1.74(\mathrm{dd}, \mathrm{J}=7.0,3.2 \mathrm{~Hz}, 3 \mathrm{H})^{* *}$. ${ }^{13} \mathrm{C}$ NMR $\left(\mathrm{CDCl}_{3}, 75 \mathrm{MHz}\right) \delta: 204.6,204.5,172.8,171.8,171.7,136.6,136.6,136.5$, $136.2,136.1,131.1,131.0,129.5,128.0,126.9,126.4,116.6,116.5,91.1,91.0,90.6,90.3$, $68.7,52.3,50.6,50.5,38.9,38.7,13.8,13.5$. IR (thin film) $v 3028,2947,1968,1743$, 1639, 1444, 1393, 1266, $1225 \mathrm{~cm}^{-1}$; MS m/z (\%) 374 (26), 344 (9), 316 (10), 284 (52), 105 (100); EI-HRMS calcd for $\mathrm{C}_{24} \mathrm{H}_{24} \mathrm{NO}_{3}\left[\mathrm{M}-1^{+}\right], \mathrm{m} / \mathrm{z}$ 374.1756, found 374.1761 ${ }^{*}$ denotes the major diastereomer; ${ }^{* *}$ denotes the minor diastereomer 


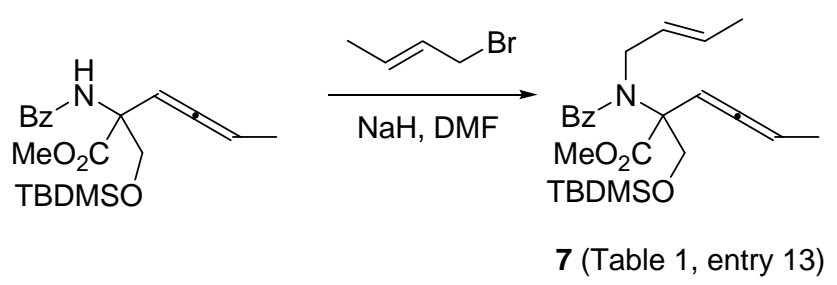

\section{2-(Benzoylbut-2-enylamino)-2-(tert-butyldimethylsilyloxymethyl)-hexa-3,4-dienoic}

acid methyl ester (7, Table 1, entry 13). Followed general procedure for N-alkylation of N-protected allenic amino acid methyl esters (page S10):

2-Benzoylamino-2-(tert-butyl-dimethyl-silanyloxymethyl)-hexa-3,4-dienoic acid methyl ester (90 mg, $0.23 \mathrm{mmol}), \mathrm{NaH}$ (60\% dispersion in mineral oil) (19 $\mathrm{mg}, 0.46 \mathrm{mmol})$, crotyl bromide (47 $\mu \mathrm{L}, 0.46 \mathrm{mmol})$.

Purification by flash chromatography (hexanes-EtOAc, $9: 1$, v/v) afforded the title compound (43 mg, 42\%).

${ }^{1} \mathrm{H}$ NMR $\left(300 \mathrm{MHz}, \mathrm{CDCl}_{3}\right)$ $\delta$ 7.43-7.27 (m, 5H), 5.65-5.43 (m, 3H), 5.35-5.29 (m, 1H), 4.51-4.45 (m, 1H), 4.10 (d, J=10.2 Hz, 1H), 4.09-4.00 (m, 1H), 3.92 (br d, J = 16.4 Hz, $1 \mathrm{H}), 3.74(\mathrm{~s}, 3 \mathrm{H}), 1.70-1.67(\mathrm{~m}, 6 \mathrm{H}), 0.89(\mathrm{~s}, 9 \mathrm{H}), 0.07(\mathrm{~s}, 6 \mathrm{H}) ;{ }^{13} \mathrm{C} \mathrm{NMR}\left(\mathrm{CDCl}_{3}, 75\right.$ MHz) $\delta 205.1,204.9,172.5,170.8,136.9,129.8,129.5,129.3,128.1,127.9,127.1$, $127.0,126.7,126.5,126.5,89.9,89.8,89.5,68.3,64.0,63.8,52.1,50.2,50.1,25.8,25.8$, $18.1,17.7,13.9,13.6,12.7,-5.5,5.6$; IR (thin film) $v 2951,1968,1743,1639,1401,1250$ $\mathrm{cm}^{-1}$; MS m/z (\%) 443 (25), 386 (43), 298 (35), 244 (32), 105 (100); EI-HRMS calcd for $\mathrm{C}_{25} \mathrm{H}_{37} \mathrm{NO}_{4} \mathrm{Si}\left[\mathrm{M}^{+}\right], \mathrm{m} / \mathrm{z}$ 443.2492, found 443.2503.

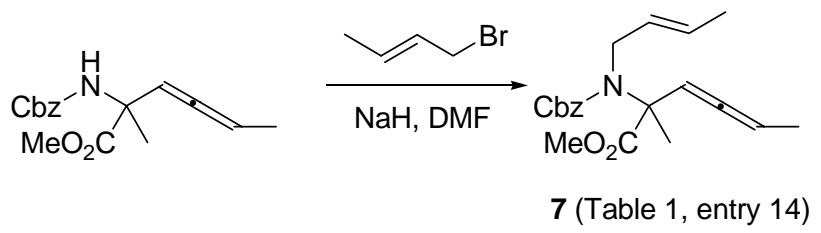


2-(Benzyloxycarbonyl-but-2-enyl-amino)-2-methyl-hexa-3,4-dienoic acid methyl ester (7, Table 1, entry 14). Followed general procedure for N-alkylation of N-protected allenic amino acid methyl esters (page S10):

2-Benzyloxycarbonylamino-2-methylhexa-3,4-dienoic acid methyl ester (66 mg, 0.23 mmol), $\mathrm{NaH}$ (60\% dispersion in mineral oil) (14 mg, $0.34 \mathrm{mmol})$, crotyl bromide $(50 \mu \mathrm{L}$, $0.45 \mathrm{mmol}$ ).

Purification by flash chromatography (hexanes-EtOAc, $9: 1$, v/v) afforded the title compound (52mg, 66\%).

${ }^{1} \mathrm{H}$ NMR (300 MHz, $\left.\mathrm{CDCl}_{3}\right) \delta:$ 7.36-7.30 (m, 5H), $5.64(\mathrm{dq}, \mathrm{J}=14.5,6.3 \mathrm{~Hz}, 1 \mathrm{H}), 5.51-$ $5.46(\mathrm{~m}, 2 \mathrm{H}), 5.39-5.30(\mathrm{~m}, 1 \mathrm{H}), 5.13(\mathrm{~s}, 2 \mathrm{H}), 3.96$ (br d, 2H), 3.67 (br s, 3H), 1.68 (dd, J $=7.0,3.1 \mathrm{~Hz}, 3 \mathrm{H}), 1.69-1.67(\mathrm{~m}, 3 \mathrm{H}), 1.52(\mathrm{~s}, 3 \mathrm{H}) ;{ }^{13} \mathrm{C} \mathrm{NMR}\left(\mathrm{CDCl}_{3}, 75 \mathrm{MHz}\right) \delta: 205.0$, $173.3,156.0,136.8,128.7,128.5,128.2,127.4,125.2,93.9,90.9,67.6,64.5,52.6,17.9$, 14.0. IR (thin film) $\vee 2949,1965,1746,1698,1450,1400,1353,1250 \mathrm{~cm}^{-1} . \mathrm{MS}$ (GC/MS) m/z $284\left[\mathrm{M}-59^{+}\right]$.

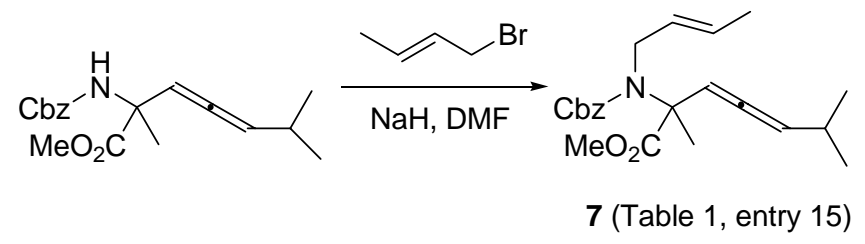

2-(Benzyloxycarbonylbut-2-enylamino)-2,6-dimethylhepta-3,4-dienoic acid methyl ester (7, Table 1, entry 15). Followed general procedure for $\mathrm{N}$-alkylation of N-protected allenic amino acid methyl esters (page S10):

2-Benzyloxycarbonylamino-2,6-dimethylhepta-3,4-dienoic acid methyl ester (60 mg, $0.189 \mathrm{mmol}), \mathrm{NaH}(60 \%$ dispersion in mineral oil) $(15 \mathrm{mg}, 0.38 \mathrm{mmol})$, crotyl bromide $(39 \mu \mathrm{L}, 0.38 \mathrm{mmol})$. 
Purification by flash chromatography (hexanes-EtOAc, $9: 1$, v/v) afforded the title compound (43 mg, 61\%).

${ }^{1} \mathrm{H}$ NMR $\left(300 \mathrm{MHz}, \mathrm{CDCl}_{3}, 323 \mathrm{~K}\right) \delta$ 7.34-7.27 (m, 5H), 5.69-5.50 (m, 3H), $5.40(\mathrm{t}, J=$ $6.1 \mathrm{~Hz}, 1 \mathrm{H}), 5.13(\mathrm{~s}, 2 \mathrm{H}), 3.98(\mathrm{~d}, J=4.1 \mathrm{~Hz}, 2 \mathrm{H}), 3.59(\mathrm{~s}, 3 \mathrm{H}), 2.41-2.29(\mathrm{~m}, 1 \mathrm{H})$, 1.1.68 (d, $J=6.2 \mathrm{~Hz}, 3 \mathrm{H}), 1.54(\mathrm{~s}, 3 \mathrm{H}), 1.04(\mathrm{~d}, J=6.7 \mathrm{~Hz}, 6 \mathrm{H})$; Two additional signals observed in the ${ }^{1} \mathrm{H}$ NMR spectra were attributed to conformational/rotational isomers due to the carbamate protecting group: $4.10(\mathrm{~d}, J=2.8 \mathrm{~Hz}), 1.61(\mathrm{~d}, J=4.3 \mathrm{~Hz})$. See spectra for detail.

${ }^{13} \mathrm{C}$ NMR $\left(\mathrm{CDCl}_{3}, 75 \mathrm{MHz}, 323 \mathrm{~K}\right) \delta 202.4,172.8,155.8,136.6,128.3,128.0,127.9$, $127.0,103.0,95.3,67.3,64.5,52.2,28.1,22.5,22.4,17.5,12.8$; IR (thin film) v 2959, 1962, 1747, 1701, $1252 \mathrm{~cm}^{-1}$; MS m/z (\%) 371 (24), 312 (25), 258 (31), 91 (100); EIHRMS calcd for $\mathrm{C}_{22} \mathrm{H}_{29} \mathrm{NO}_{4}\left[\mathrm{M}^{+}\right], \mathrm{m} / \mathrm{z}$ 371.2097, found 371.2104.

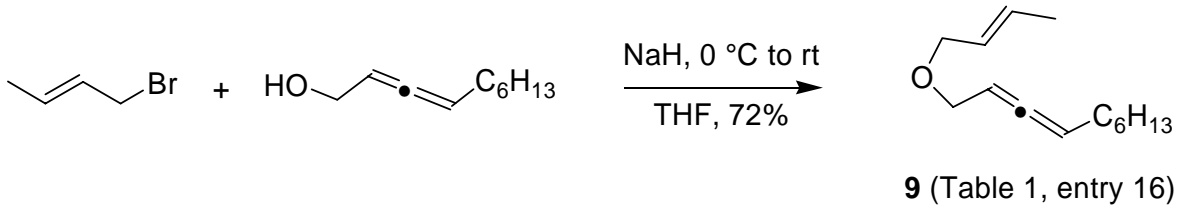

1-[(E)-2-Butenyloxy]-2,3-decadiene (9, Table 1, entry 16): 2,3-Decadien-1-ol (202 mg, $1.30 \mathrm{mmol}, 1.00$ eq.) was added via a syringe into a suspension of $\mathrm{NaH}$ (70 mg, 2.59 mmol, 2.00 eq.) in dry THF $(20 \mathrm{~mL})$. The mixture was stirred under $\mathrm{N}_{2}$ at $\mathrm{rt}$ for 40 min, and crotyl bromide (Aldrich $85 \%$ tech. grade, $175 \mu \mathrm{L}, 1.43 \mathrm{mmol}, 1.10$ eq.) was added followed by HMPA ( $47 \mu \mathrm{L}, 0.26 \mathrm{mmol}, 0.20$ eq.). The resulting suspension was allowed to react for $20 \mathrm{~h}$ at rt. The reaction was then quenched with sat. $\mathrm{NH}_{4} \mathrm{Cl}(10 \mathrm{~mL})$, diluted with ether $(50 \mathrm{~mL})$, washed with water $(5 \mathrm{~mL})$ and brine $(2 \times 5 \mathrm{~mL})$, dired $\left(\mathrm{MgSO}_{4}\right)$, filtered and concentrated. Flash chromatography $\left(\mathrm{SiO}_{2}\right.$, hexanes : ethyl acetate $=50: 1)$ of the residue affords the title compound as a colorless oil $(198 \mathrm{mg}, 72 \%):{ }^{1} \mathrm{H}$ 
NMR (300 MHz, $\left.\mathrm{CDCl}_{3}\right) \delta 5.77-5.50(\mathrm{~m}, 2 \mathrm{H}), 5.20-5.14(\mathrm{~m}, 2 \mathrm{H})$, 4.01-3.90 (m, 4H), 2.05-1.97 (m, 2H), $1.72(\mathrm{~d}, J=6.2 \mathrm{~Hz}, 3 \mathrm{H}), 1.43-1.24(\mathrm{~m}, 8 \mathrm{H}), 0.89$ (t, $J=6.3 \mathrm{~Hz}, 3 \mathrm{H})$; ${ }^{13} \mathrm{C}$ NMR $\left(75 \mathrm{MHz}, \mathrm{CDCl}_{3}\right) \delta 205.0,129.4,127.6,91.8,88.5,70.4,68.4,31.7,29.1,28.7$, 28.6, 22.6, 17.7, 14.1; IR (neat) $\vee 1963 \mathrm{~cm}^{-1}$; EI-HRMS calcd for $\mathrm{C}_{14} \mathrm{H}_{24} \mathrm{O}\left[\mathrm{M}^{+}\right] \mathrm{m} / \mathrm{z}$ 208.1827 , found 208.1825 .

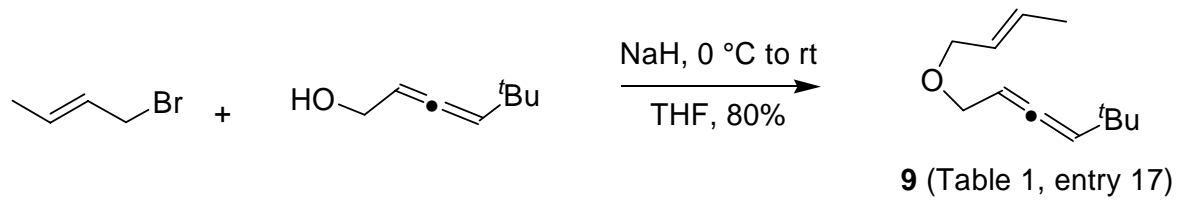

1-[(E)-2-Butenyloxy]-5,5-dimethyl-2,3-hexadiene (9, Table 1, entry 17): This compound was prepared following the procedure for the preparation of the preceding compound from 5,5-dimethyl-2,3-hexadiene-1-ol (103 mg, $0.82 \mathrm{mmol}, 1.0$ eq.), $\mathrm{NaH}$ (65 $\mathrm{mg}, 65 \%$ dispersion in mineral oil, $1.6 \mathrm{mmol}, 2.0$ eq.), crotyl bromide $(125 \mu \mathrm{L}, 1.0 \mathrm{mmol}$, 1.3 eq.), and HMPA (30 $\mu \mathrm{L}, 0.16 \mathrm{mmol}, 0.20$ eq.) as a colorless oil $(118 \mathrm{mg}, 80 \%):{ }^{1} \mathrm{H}$ NMR (300 MHz, $\left.\mathrm{CDCl}_{3}\right) \delta 5.73(\mathrm{dtt}, J=1.1,6.3,15.3 \mathrm{~Hz}, 1 \mathrm{H}), 5.59(\mathrm{dtq}, J=1.4,5.9$, $15.3 \mathrm{~Hz}, 1 \mathrm{H}), 3.98(\mathrm{dd}, \mathrm{J}=2.4,6.8 \mathrm{~Hz}, 2 \mathrm{H}), 3.93(\mathrm{dq}, \mathrm{J}=1.2,6.1 \mathrm{~Hz}, 2 \mathrm{H}), 1.72(\mathrm{dd}, J=$ 2.4, $6.2 \mathrm{~Hz}, 3 \mathrm{H}), 1.05$ (s, 9H); ${ }^{13} \mathrm{C} \mathrm{NMR}\left(75 \mathrm{MHz}, \mathrm{CDCl}_{3}\right) \delta 202.3,129.3,127.7,103.8$, 90.6, 70.4, 68.6, 41.0, 30.2, 17.6; IR (neat) $v 1961 \mathrm{~cm}^{-1}$; EI-HRMS calcd for $\mathrm{C}_{12} \mathrm{H}_{20} \mathrm{O}$ $\left[\mathrm{M}^{+}\right] \mathrm{m} / \mathrm{z}$ 180.1514, found 180.1513 .

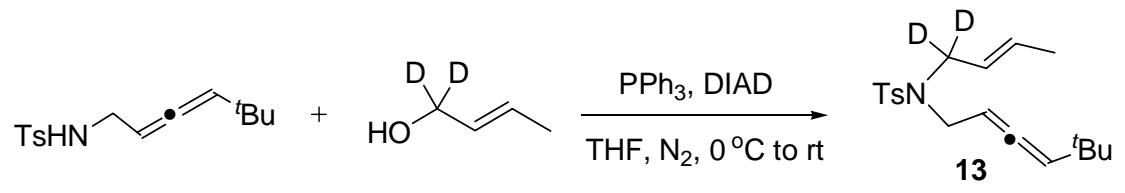

$N$-[2-(E)-butenyl-1,1-d $]$ - $N$-(5,5-dimethyl-2,3-hexadienyl)-4-methylbenzenesulfona-

mide (13). The general procedure for the Mitsunobu reaction (page S5) was followed using $\quad \mathrm{PPh}_{3} \quad(119 \quad \mathrm{mg}, \quad 0.452 \quad \mathrm{mmol}), \quad \mathrm{N}-(5,5$-Dimethyl-2,3-hexadienyl)-4- 
methylbenzenesulfonamide (112 mg, $0.401 \mathrm{mmol}), 1,1$-dideutero-2-(E)-buten-1-ol (32 $\mathrm{mg}, 0.43 \mathrm{mmol})$, and DIAD $(89 \mu \mathrm{L}, 0.45 \mathrm{mmol})$. The resulting residue was purified by silica gel chromatography (ethyl acetate-hexanes 1:19-1:7) to afford $82 \mathrm{mg}$ of ene-allene 13 as a colorless oil in $61 \%$ yield. ${ }^{1} \mathrm{H}$ NMR $\left(300 \mathrm{MHz}, \mathrm{CDCl}_{3}\right) \delta: 7.70(1 / 2 \mathrm{AB} \mathrm{q}, J=8.3$ $\mathrm{Hz}, 2 \mathrm{H}), 7.29(1 / 2 \mathrm{AB} \mathrm{q}, J=8.3 \mathrm{~Hz}, 2 \mathrm{H}), 5.60(\mathrm{dq}, J=15.2,6.5 \mathrm{~Hz}, 1 \mathrm{H}), 5.27$ (bd, $J=$ $15.2 \mathrm{~Hz}, 1 \mathrm{H}), 5.13(\mathrm{dt}, J=6.2,2.3 \mathrm{~Hz}, 1 \mathrm{H}), 4.95(\mathrm{q}, J=6.6 \mathrm{~Hz}, 1 \mathrm{H}), 3.85(\mathrm{ddd}, J=15.0$, 6.6, $2.4 \mathrm{~Hz}, 1 \mathrm{H}), 3.77(\mathrm{ddd}, J=15.0,7.2,2.4 \mathrm{~Hz}, 1 \mathrm{H}), 2.43(\mathrm{~s}, 3 \mathrm{H}), 1.64(\mathrm{dd}, J=6.5,1.5$ $\mathrm{Hz}, 3 \mathrm{H}), 1.01(\mathrm{~s}, 9 \mathrm{H}) ;{ }^{13} \mathrm{C} \mathrm{NMR}\left(75 \mathrm{MHz}, \mathrm{CDCl}_{3}\right) \delta: 202.6,143.1,138.0,130.7,129.7$, 127.3, 125.3, 104.6, 88.6, 46.2, 31.9, 30.2, 21.6, 17.8; IR (neat) 2960, 2927, 2863, 1957, 1593, 1455, $1346 \mathrm{~cm}^{-1}$; MS m/z (\%) 335 (12), 296 (41), 240 (54), 184 (100), 155 (81), 111 (21), 91 (72) 57 (77); EI-HRMS calcd for $\mathrm{C}_{19} \mathrm{H}_{25} \mathrm{D}_{2} \mathrm{NO}_{2} \mathrm{~S}$ : 335.1888; found: 335.1874 .

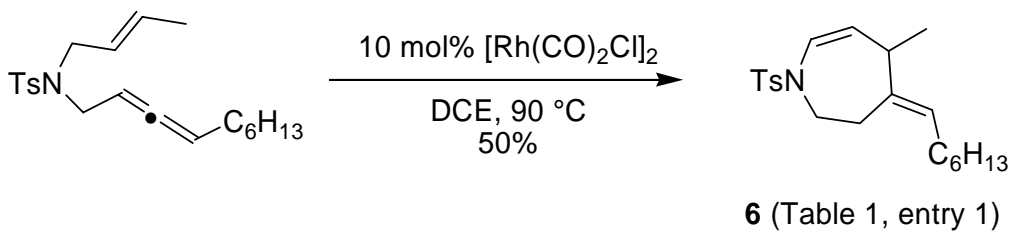

\section{4-(E)-Heptylidene-5-methyl-1-(4-methylbenzenesulfonyl)-2,3,4,5-tetrahydro-}

1H-azepine (6, Table 1, entry 1): To a flame-dried test tube equipped with a magnetic stirring bar was added $N$-[(E)-2-butenyl]- $N$-2,3-decadienyl-4-methylbenzenesulfonamide (5, Table 1 , entry $1,36.2 \mathrm{mg}, 0.10 \mathrm{mmol})$ and 1,2-dichloroethane $(0.5 \mathrm{~mL})$. The test tube was evacuated and charged with $\mathrm{N}_{2} 3$ times and $\left[\mathrm{Rh}(\mathrm{CO})_{2} \mathrm{Cl}\right]_{2}(3.9 \mathrm{mg}, 0.010 \mathrm{mmol})$ was added. The mixture was stirred and heated at $90{ }^{\circ} \mathrm{C}$ for $2.5 \mathrm{~h}$ under $\mathrm{N}_{2}$ atmosphere (1 atm) and was monitored by GC until the starting material was consumed. The solvent was removed in vacuo and the residue was purified by flash chromatography $\left(\mathrm{SiO}_{2}\right.$, hexane:ethyl acetate $=9: 1)$ to afford the title compound as a colorless oil $(18.0 \mathrm{mg}, 50 \%)$ : 
${ }^{1} \mathrm{H}$ NMR $\left(300 \mathrm{MHz}, \mathrm{CDCl}_{3}\right) \delta$ 7.72-7.68 (m, 2H), $7.29(\mathrm{~d}, J=8.1 \mathrm{~Hz}, 2 \mathrm{H}), 6.37(\mathrm{dd}, J=$ 1.7, $9.2 \mathrm{~Hz}, 1 \mathrm{H}), 5.12(\mathrm{dt}, J=1.0,7.0 \mathrm{~Hz}, 1 \mathrm{H}), 4.82(\mathrm{dd}, J=5.0,9.2 \mathrm{~Hz}, 1 \mathrm{H}), 3.66-3.61$ (m, 2H), 3.10-3.01 (m, 1H), 2.46-2.42 (m, 2H), $2.42(\mathrm{~s}, 3 \mathrm{H}), 1.79-1.74(\mathrm{~m}, 2 \mathrm{H}), 1.33-$ $1.20(\mathrm{~m}, 8 \mathrm{H}), 1.10(\mathrm{~d}, J=7.2 \mathrm{~Hz}, 3 \mathrm{H}), 0.88(\mathrm{t}, J=7.0 \mathrm{~Hz}, 3 \mathrm{H}) ;{ }^{13} \mathrm{C} \mathrm{NMR}(75 \mathrm{MHz}$, $\left.\mathrm{CDCl}_{3}\right) \delta 143.3,138.5,129.6,127.2,126.9,125.8,125.0,119.0,47.6,39.4,31.7,29.6$, 29.02, 28.97, 27.4, 22.6, 21.5, 20.6, 14.0; IR (neat) $\vee 2955,2926,1347,1163 \mathrm{~cm}^{-1}$; EIHRMS calcd for $\mathrm{C}_{21} \mathrm{H}_{31} \mathrm{NO}_{2} \mathrm{~S}\left[\mathrm{M}^{+}\right] \mathrm{m} / \mathrm{z}$ 361.2076, found 361.2085.
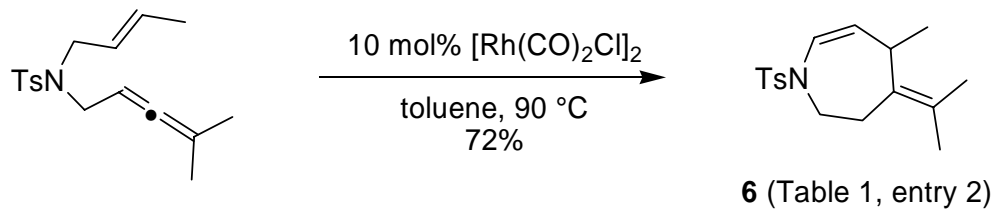

\section{4-Isopropylidene-5-methyl-1-(4-methylbenzenesulfonyl)-2,3,4,5-tetrahydro-}

$1 \mathrm{H}$-azepine (6, Table 1, entry 2): This compound was prepared following the procedure for the preparation of the preceding compound from $N$-[(E)-2-butenyl]- $N-2,3-$ pentadienyl-4-methylbenzene-sulfonamide (5, Table 1, entry 2, $22.5 \mathrm{mg}, 0.074 \mathrm{mmol})$ and $\left[\mathrm{Rh}(\mathrm{CO})_{2} \mathrm{Cl}\right]_{2}(2.8 \mathrm{mg}, 0.0074 \mathrm{mmol})$ in toluene $(0.5 \mathrm{~mL})$ under $\mathrm{N}_{2}$ atmosphere in $2 \mathrm{~h}$ as a colorless oil $(16.1 \mathrm{mg}, 72 \%):{ }^{1} \mathrm{H} \mathrm{NMR}\left(300 \mathrm{MHz}, \mathrm{CDCl}_{3}\right) \delta$ 7.68-7.65 (m, 2H), 7.28 (d, $J=8.0 \mathrm{~Hz}, 2 \mathrm{H}), 6.34(\mathrm{dd}, J=1.6,9.4 \mathrm{~Hz}, 1 \mathrm{H}), 4.90(\mathrm{dd}, J=5.5,9.4 \mathrm{~Hz}, 1 \mathrm{H}), 3.65$ (ddd, $J=3.9,7.7,12.6 \mathrm{~Hz}, 1 \mathrm{H}), 3.56-3.34(\mathrm{~m}, 2 \mathrm{H}), 2.68-2.56(\mathrm{~m}, 1 \mathrm{H}), 2.42(\mathrm{~s}, 3 \mathrm{H}), 2.23-$ 2.09 (m, 1H), 1.53 (s, 3H), 1.49 (s, 3H), 1.01 (d, $J=7.3 \mathrm{~Hz}, 3 \mathrm{H}) ;{ }^{13} \mathrm{C}$ NMR $(75 \mathrm{MHz}$, $\left.\mathrm{CDCl}_{3}\right) \delta 143.2,136.6,131.6,129.5,126.9,125.2,124.5,120.2,48.8,35.8,27.3,21.5$, 21.1, 20.3, 19.8; IR (neat) $v$ 2959, 2924, 1650, 1597, 1344, $1164 \mathrm{~cm}^{-1}$; EI-HRMS calcd for $\mathrm{C}_{17} \mathrm{H}_{23} \mathrm{NO}_{2} \mathrm{~S}\left[\mathrm{M}^{+}\right] \mathrm{m} / \mathrm{z} 305.1449$, found 305.1450 . 

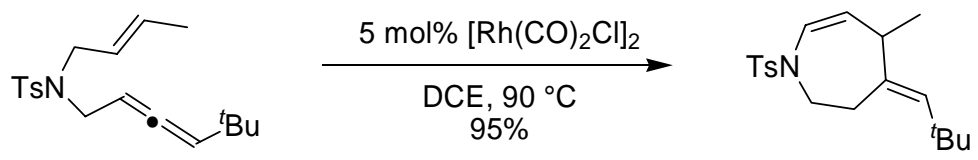

6 (Table 1, entry 3)

\section{4-[(E)-2,2-Dimethylpropylidene]-5-methyl-1-(4-methylbenzenesulfonyl)-}

2,3,4,5-tetrahydro-1H-azepine (6, Table 1, entry 3): This compound was prepared following the procedure for the preparation of 6 (Table 1, entry 1) from $N-[(E)-2-$ butenyl]- $N$-(5,5-dimethyl-2,3-hexadienyl)-4-methylbenzenesulfonamide $\quad$ (5, Table 1, entry 3, $29.9 \mathrm{mg}, 0.090 \mathrm{mmol})$ and $\left[\mathrm{Rh}(\mathrm{CO})_{2} \mathrm{Cl}\right]_{2}(1.7 \mathrm{mg}, 0.0045 \mathrm{mmol})$ in 1,2dichloroethane $(0.5 \mathrm{~mL})$ for $1.5 \mathrm{~h}$ under Ar atmosphere (1 atm) as a colorless oil (28.3 mg, 95\%): ${ }^{1} \mathrm{H}$ NMR $\left(300 \mathrm{MHz}, \mathrm{CDCl}_{3}\right) \delta$ 7.72-7.68 (m, 2H), 7.32-7.29 (m, 2H), 6.35 $(\mathrm{dd}, J=1.5,9.3 \mathrm{~Hz}, 1 \mathrm{H}), 5.21(\mathrm{~d}, J=1.0 \mathrm{~Hz}, 1 \mathrm{H}), 4.83(\mathrm{dd}, J=5.5,9.3 \mathrm{~Hz}, 1 \mathrm{H}), 3.62$ (ddd, $J=3.8,7.7,13.5 \mathrm{~Hz}, 1 \mathrm{H}), 3.52(\mathrm{ddd}, J=3.8,7.7,13.5 \mathrm{~Hz}, 1 \mathrm{H}), 2.98(\mathrm{dtq}, J=1.1$, 7.0, 7.0 Hz, 1H), 2.73 (dddd, $J=1.1,3.8,7.7,15.5 \mathrm{~Hz}, 1 \mathrm{H}), 2.55$ (dddd, $J=1.1,3.8,7.7$, $15.5 \mathrm{~Hz}, 1 \mathrm{H}), 2.42$ (s, 3H), 1.07 (d, J=7.2 Hz, 3H), $1.04(\mathrm{~s}, 9 \mathrm{H}) ;{ }^{13} \mathrm{C}$ NMR $(75 \mathrm{MHz}$, $\left.\mathrm{CDCl}_{3}\right) \delta 143.5,137.8,136.4,135.3,129.7,126.9,125.7,119.1,47.8,41.0,31.9,31.2$, 29.3, 21.5, 21.1; IR (neat) $\vee 2958,1647,1350,1164 \mathrm{~cm}^{-1}$; EI-HRMS calcd for $\mathrm{C}_{19} \mathrm{H}_{27} \mathrm{NO}_{2} \mathrm{~S}\left[\mathrm{M}^{+}\right] \mathrm{m} / \mathrm{z} 333.1763$, found 333.1777.

The stereochemistry of azepine 6 (Table 1, entry 3) was confirmed by ${ }^{1} \mathrm{H}$ NOE study as illustrated below.

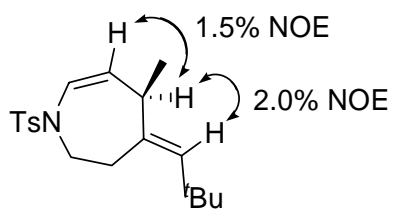




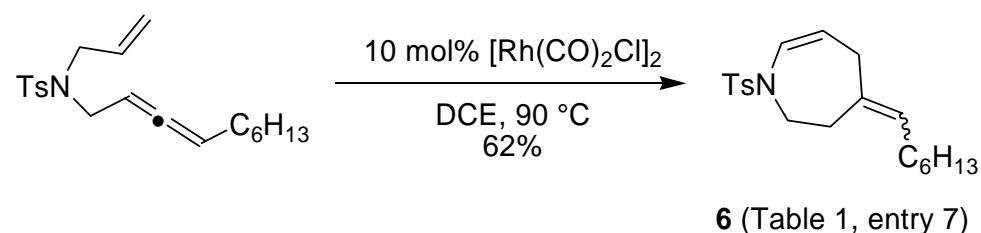

4-Heptylidene-1-(4-methylbenzenesulfonyl)-2,3,4,5-tetrahydro-1H-azepine (6, Table 1, entry 7): This compound was prepared following the procedure for the preparation of $\mathbf{6}$ (Table 1, entry 1) from $\mathrm{N}$-allyl- $N$-2,3-decadienyl-4methylbenzenesulfonamide (5, Table 1 , entry $7,40.8 \mathrm{mg}, 1.20 \mathrm{mmol})$ and $\left[\mathrm{Rh}(\mathrm{CO})_{2} \mathrm{Cl}\right]_{2}$ (4.6 mg, $0.012 \mathrm{mmol})$ in 1,2-dichloroethane $(0.6 \mathrm{~mL})$ for $4 \mathrm{~h}$ under Ar atmosphere (1 atm) as a colorless oil $(25.1 \mathrm{mg}, 62 \%)$ as a inseparable $1.3: 1$ mixture of the two geometric isomers of the external double bond.: ${ }^{1} \mathrm{H}$ NMR $\left(300 \mathrm{MHz}, \mathrm{CDCl}_{3}\right) \delta 7.71-7.68$ $(\mathrm{m}, 2 \mathrm{H}), 7.30-7.27(\mathrm{~m}, 2 \mathrm{H}), 6.45-6.40(\mathrm{~m}, 1 \mathrm{H}), 5.16-5.12(\mathrm{~m}, 0.56 \mathrm{H}), 5.05(\mathrm{dt}, J=5.5$, 8.7 Hz, 0.44H), $4.98(\mathrm{dt}, J=5.5,9.0 \mathrm{~Hz}, 0.56 \mathrm{H}), 4.84-4.80(\mathrm{~m}, 0.44 \mathrm{H}), 3.72-3.69(\mathrm{~m}$, 2H), 2.82-2.75 (m, 2H), $2.43(\mathrm{~s}, 3 \mathrm{H}), 2.50-2.36(\mathrm{~m}, 2 \mathrm{H}), 1.85-1.81(\mathrm{~m}, 1 \mathrm{H}), 1.74-1.72$ $(\mathrm{m}, 1 \mathrm{H}), 1.37-1.11(\mathrm{~m}, 8 \mathrm{H}), 0.89(\mathrm{t}, J=6.8 \mathrm{~Hz}, 3 \mathrm{H})$; IR (neat) $v 2954,2926,1345,1162$ $\mathrm{cm}^{-1}$
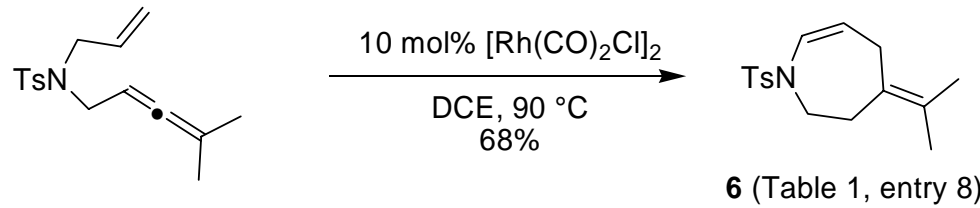

\section{4-Isopropylidene-1-(4-methylbenzenesulfonyl)-2,3,4,5-tetrahydro-1H-azepine}

(6, Table 1, entry 8): This compound was prepared following the procedure for the preparation of 6 (Table 1, entry 1) from $N$-allyl-4-methyl- $N$-(4-methyl-2,3-pentadienyl)benzenesulfonamide $(5$, Table 1 , entry $8,35.5 \mathrm{mg}, 0.12 \mathrm{mmol})$ and $\left[\mathrm{Rh}(\mathrm{CO})_{2} \mathrm{Cl}\right]_{2}(4.7$ $\mathrm{mg}, 0.012 \mathrm{mmol})$ in 1,2-dichloroethane $(0.5 \mathrm{~mL})$ for $6 \mathrm{~h}$ under $\mathrm{N}_{2}$ atmosphere $(1 \mathrm{~atm})$ as a colorless oil $(24.0 \mathrm{mg}, 68 \%):{ }^{1} \mathrm{H}$ NMR $\left(300 \mathrm{MHz}, \mathrm{CDCl}_{3}\right) \delta 7.68(\mathrm{~d}, J=8.2 \mathrm{~Hz}, 2 \mathrm{H})$, 
$7.28(\mathrm{~d}, J=8.2 \mathrm{~Hz}, 2 \mathrm{H}), 6.43(\mathrm{~d}, J=8.9 \mathrm{~Hz}, 1 \mathrm{H}), 5.02(\mathrm{dt}, J=5.6,8.9 \mathrm{~Hz}, 1 \mathrm{H}), 3.73(\mathrm{t}, J$ $=5.9 \mathrm{~Hz}, 1 \mathrm{H}), 2.83(\mathrm{~d}$, broaden, $J=5.4 \mathrm{~Hz}, 2 \mathrm{H}), 2.42(\mathrm{~s}, 3 \mathrm{H}), 2.51-2.35(\mathrm{~m}, 2 \mathrm{H}), 1.57(\mathrm{~s}$, 3H), $1.38(\mathrm{~s}, 3 \mathrm{H}) ;{ }^{13} \mathrm{C} \mathrm{NMR}\left(75 \mathrm{MHz}, \mathrm{CDCl}_{3}\right) \delta 143.3,137.1,129.5,127.9,127.6,126.8$, 125.9, 111.7, 47.1, 32.2, 28.3, 21.5, 20.4, 19.9; IR (neat) v 2921, 1648, 1339, $1162 \mathrm{~cm}^{-1}$; EI-HRMS calcd for $\mathrm{C}_{16} \mathrm{H}_{21} \mathrm{NO}_{2} \mathrm{~S}\left[\mathrm{M}^{+}\right] \mathrm{m} / \mathrm{z}$ 291.1293, found 291.1292.

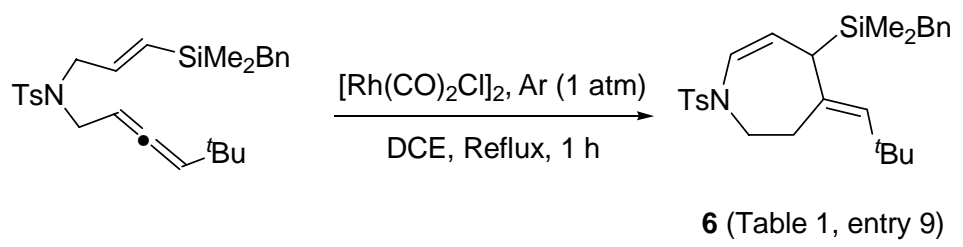

\section{4-(E)-(2,2-Dimethyl-propylidene)-1-(4-methylbenzenesulfonyl)-5-benzyldimethylsi-}

lyl-2,3,4,5-tetrahydro-1H-azepine (6, Table 1, entry 9). Ene-allene 5 (Table 1, entry 9) (53 mg, $0.11 \mathrm{mmol})$ was dissolved in dichloroethane $(570 \mu \mathrm{L})$ under Ar atmosphere and degassed (2x) via freeze-pump-thaw method followed by addition of $\left[\mathrm{Rh}(\mathrm{CO})_{2} \mathrm{Cl}\right]_{2}(4.0$ $\mathrm{mg}, 0.010 \mathrm{mmol})$. The resulting solution was allowed to react at $90{ }^{\circ} \mathrm{C}$ for $1 \mathrm{~h}$. Reaction progress was monitored by TLC (elution with benzene). Upon complete consumption of starting material the solution was diluted with hexanes $(1.5 \mathrm{~mL})$ and purified by silica gel chromatography (ethyl acetate-hexanes 1:19-1:7) to afford $51 \mathrm{mg}$ of title compound as a colorless oil in 97\% yield. ${ }^{1} \mathrm{H}$ NMR $\left(300 \mathrm{MHz}, \mathrm{C}_{6} \mathrm{D}_{6}\right) \delta: 7.72(1 / 2 \mathrm{AB} \mathrm{q}, J=8.2 \mathrm{~Hz}$, 2H), 7.13-7.10 (m, 2H), 7.02-6.97 (m, 1H), 6.90-6.87 (m, 2H), $6.78(\mathrm{~d}, J=10.5 \mathrm{~Hz}, 1 \mathrm{H})$, $6.74(1 / 2 \mathrm{AB}$ q, $J=8.2 \mathrm{~Hz}, 2 \mathrm{H}), 4.97(\mathrm{~s}, 1 \mathrm{H}), 4.68(\mathrm{dd}, J=10.2,6.7 \mathrm{~Hz}, 1 \mathrm{H}), 4.31$ (dd, $J$ $=13.0,7.6 \mathrm{~Hz}, 1 \mathrm{H}), 2.84(\mathrm{dd}, J=14.7,7.6 \mathrm{~Hz}, 1 \mathrm{H}), 2.72(\mathrm{dd}, J=13.0,9.8 \mathrm{~Hz}, 1 \mathrm{H}), 2.59$ (d, $J=6.7 \mathrm{~Hz}, 1 \mathrm{H}), 1.89$ (d, $J=8.2 \mathrm{~Hz}, 2 \mathrm{H}), 1.82(\mathrm{~s}, 3 \mathrm{H}), 1.75(\mathrm{dd}, J=14.6,9.1 \mathrm{~Hz}, 1 \mathrm{H})$,

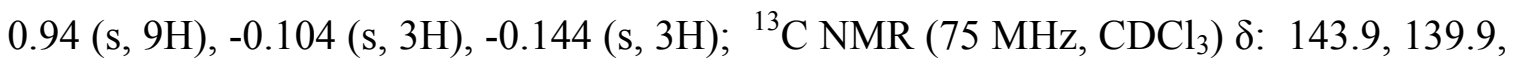
$136.7,136.3,135.4,130.1,128.5,127.2,124.5,123.5,112.1,49.2,44.4,32.4,32.1,31.1$, 
30.0, 25.0, 21.7, -3.45, -3.49; IR (neat) 3058, 3022, 2950, 2925, 2899, 2963, 2361, 1650, 1598, 1496, 1352, $1168 \mathrm{~cm}^{-1} ;$ MS m/z (\%) 467 (27), 452 (24), 410 (69), 376 (47), 312 (34), 213 (48), 149 (100); EI-HRMS calcd for $\mathrm{C}_{27} \mathrm{H}_{37} \mathrm{NO}_{2} \mathrm{SiS}$ : 467.2318; found: 467.2314.

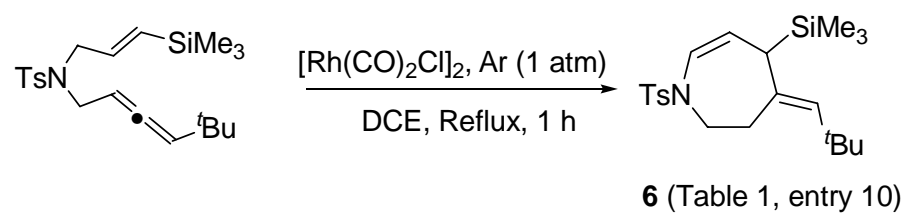

\section{4-(E)-(2,2-Dimethyl-propylidene)-1-(4-methylbenzenesulfonyl)-5-trimethylsilyl-}

2,3,4,5-tetrahydro-1H-azepine (6, Table 1, entry 10$)$. The procedure for preparation of

6 (Table 1, entry 1) was followed using ene-allene 5 (Table 1, entry 10) (58 mg, 0.15 mmol), dichloroethane $(740 \mu \mathrm{L})$, and $\left[\mathrm{Rh}(\mathrm{CO})_{2} \mathrm{Cl}\right]_{2}(5.7 \mathrm{mg}, 0.015 \mathrm{mmol})$. The resulting solution was allowed to react at $90{ }^{\circ} \mathrm{C}$ for $1 \mathrm{~h}$. Reaction progress was monitored by gas chromatography (Column temperature: $225{ }^{\circ} \mathrm{C}$; injector temperature: $250{ }^{\circ} \mathrm{C}$; detector temperature: $250{ }^{\circ} \mathrm{C}$ ). Upon complete consumption of starting material the solution was diluted with hexanes $(1.5 \mathrm{~mL})$ and purified by silica gel chromatography (ethyl acetatehexanes 1:19) to afford $47 \mathrm{mg}$ of the title compound as a colorless oil in $82 \%$ yield. ${ }^{1} \mathrm{H}$ NMR $\left(300 \mathrm{MHz}, \mathrm{CDCl}_{3}\right) \delta: 7.69(1 / 2 \mathrm{AB} \mathrm{q}, J=8.2 \mathrm{~Hz}, 2 \mathrm{H}), 7.30(1 / 2 \mathrm{AB}$ q, $J=8.2 \mathrm{~Hz}$, 2H), $6.45(\mathrm{~d}, J=10.1 \mathrm{~Hz}, 1 \mathrm{H}), 5.08(\mathrm{~s}, 1 \mathrm{H}), 4.83(\mathrm{dd}, J=10.2,6.6 \mathrm{~Hz}, 1 \mathrm{H}), 4.18-4.09$ $(\mathrm{m}, 2 \mathrm{H}), 3.05(\mathrm{dd}, J=14.8,7.6 \mathrm{~Hz}, 1 \mathrm{H}), 2.69(\mathrm{dd}, J=12.9,9.8 \mathrm{~Hz}, 1 \mathrm{H}), 2.49(\mathrm{~d}, J=6.6$ $\mathrm{Hz}, 1 \mathrm{H}), 2.42(\mathrm{~s}, 3 \mathrm{H}), 1.83(\mathrm{dd}, J=14.6,7.4 \mathrm{~Hz}, 1 \mathrm{H}), 1.05(\mathrm{~s}, 9 \mathrm{H}), 0.024(\mathrm{~s}, 9 \mathrm{H}) ;{ }^{13} \mathrm{C}$ NMR (75 MHz, $\left.\mathrm{CDCl}_{3}\right) \delta: 143.7,136.6,134.8,130.0,128.6,127.2,123.0,112.3,49.2$, $45.7,32.3,32.1,30.8,21.8,-1.58$; IR (neat) 2955, 2866, 1650, 1598, 1465, $1349 \mathrm{~cm}^{-1}$; MS m/z (\%) 391 (23), 376 (24), 335 (35) 334 (58), 236 (66), 163 (70), 91 (63), 73 (100); EI-HRMS calcd for $\mathrm{C}_{21} \mathrm{H}_{33} \mathrm{NO}_{2} \mathrm{SiS}$ : 391.2001; found: 391.2014 . 
General procedure for the rhodium catalyzed azepine formation by cycloisomerization of amino acid tethered ene-allenes.
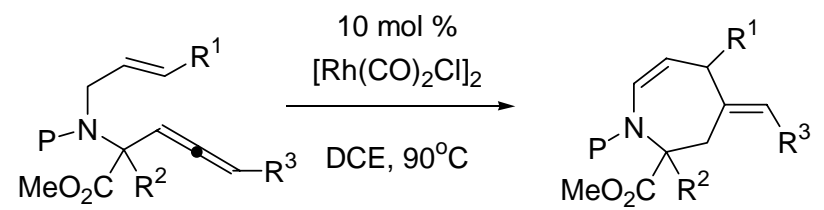

To a solution of alkenyl allene $(0.3 \mathrm{mmol})$ in DCE $(3 \mathrm{~mL})$ in a flame dried test tube, was added $\left[\mathrm{Rh}(\mathrm{CO})_{2} \mathrm{Cl}\right]_{2}(0.015 \mathrm{mmol})$ at room temperature or $70{ }^{\circ} \mathrm{C}$ under Ar atmosphere. The temperature of the oil bath was then raised to $90{ }^{\circ} \mathrm{C}$ and the reaction was followed by TLC. After 2-4 h TLC indicated no observable changes in the reaction and then another portion of $\left[\mathrm{Rh}(\mathrm{CO})_{2} \mathrm{Cl}\right](0.015 \mathrm{mmol})$ was added. Stirring was continued for additional 2 $\mathrm{h}$ (or until TLC indicated absence of starting material). The reaction mixture was then cooled to rt, and filtered through a pad of silica gel eluting with (hexanes-EtOAc, $9: 1$, $\mathrm{v} / \mathrm{v}$ ). Additional purification by flash chromatography (hexanes-EtOAc) was performed in some cases if necessary.

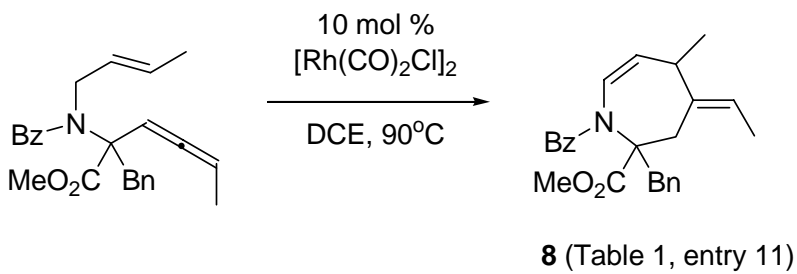

\section{1-Benzoyl-2-benzyl-4-ethylidene-5-methyl-2,3,4,5-tetrahydro-1H-azepine-2-}

carboxylic acid methyl ester (8, Table 1, entry 11). Followed general procedure for the rhodium catalyzed azepine formation by cycloisomerization of ene-allenes (page S22): 2-(Benzoyl-but-2-enylamino)-2-benzylhexa-3,4-dienoic acid methyl ester (30 mg, 0.077 $\mathrm{mmol}),\left[\mathrm{Rh}(\mathrm{CO})_{2} \mathrm{Cl}\right]_{2}$ in two portions $(2 \times 2 \mathrm{mg}, 0.01 \mathrm{mmol})$. First portion of $\left[\mathrm{Rh}(\mathrm{CO})_{2} \mathrm{Cl}\right]_{2}$ was added at $\mathrm{rt}$. The total reaction time was $4 \mathrm{~h}$. The reaction mixture was 
then cooled to rt, and applied to a pad of silica gel and eluted (hexanes-Et $\mathrm{t}_{2} \mathrm{O}, 1: 1, \mathrm{v} / \mathrm{v}$ ) to afford the title compound (30 mg, > 95\%).

${ }^{1} \mathrm{H}$ NMR (300 MHz, $\mathrm{CDCl}_{3}$ ), $8:$ 7.57-7.54 (m, 2H), 7.42-7.35 (m, 3H), 7.23-7.19 (m, 5H), $5.52(\mathrm{q}, J=6.8 \mathrm{~Hz}, 1 \mathrm{H}), 5.03(\mathrm{dd}, J=8.1,2.5,1 \mathrm{H}), 4.53(\mathrm{dd}, J=8.1,4.1 \mathrm{~Hz}, 1 \mathrm{H}), 4.20$ $(1 / 2 \mathrm{AB}, J=13.6 \mathrm{~Hz}, 1 \mathrm{H}), 3.75(\mathrm{~s}, 3 \mathrm{H}), 3.40($ br s, $1 \mathrm{H}), 3.14(1 / 2 \mathrm{AB}, J=13.6 \mathrm{~Hz}, 1 \mathrm{H})$, $2.83(1 / 2 \mathrm{AB}, J=14.6 \mathrm{~Hz}, 1 \mathrm{H}), 2.66(1 / 2 \mathrm{AB}, 14.6 \mathrm{~Hz}, 1 \mathrm{H}), 1.63(\mathrm{~d}, J=6.8 \mathrm{~Hz}, 3 \mathrm{H})$, $1.08(\mathrm{~d}, J=7.1 \mathrm{~Hz}, 1 \mathrm{H}) ;{ }^{13} \mathrm{C} \mathrm{NMR}\left(\mathrm{CDCl}_{3}, 75 \mathrm{MHz}\right) \delta: 172.7,171.4,137.0,136.4$, $135.1,130.9,130.4,128.4,128.1,128.0,127.8,126.8,125.7,121.9,69.0,52.5,40.4$, 36.9, 34.7, 21.4, 13.6. IR (thin film) v 2950, 1736, 1637, 1446, 1350, $1223 \mathrm{~cm}^{-1} ; \mathrm{MS} \mathrm{m} / \mathrm{z}$ (\%) 389 (38), 358 (15), 330 (42), 298 (42), 105 (100); EI-HRMS calcd for $\mathrm{C}_{25} \mathrm{H}_{27} \mathrm{NO}_{3}$ $\left[\mathrm{M}^{+}\right], m / z 389.1991$ found 389.1984 .

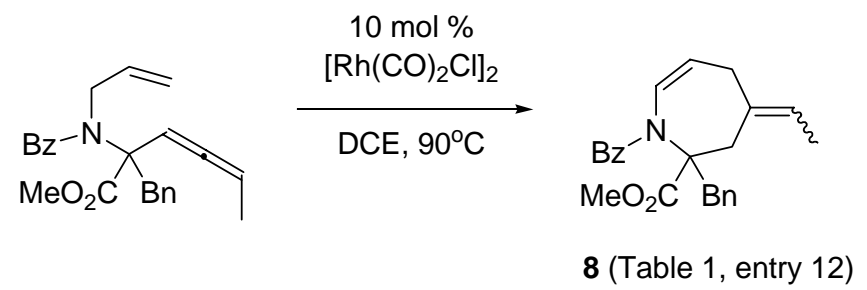

1-Benzoyl-2-benzyl-4-ethylidene-2,3,4,5-tetrahydro- $1 \mathrm{H}$-azepine-2-carboxylic acid methyl ester (8, Table 1, entry 12). Followed general procedure for the rhodium catalyzed azepine formation by cycloisomerization of ene-allenes (page S22):

2-(Allylbenzoylamino)-2-benzylhexa-3,4-dienoic acid methyl ester (120 mg, $0.32 \mathrm{mmol}$ ), $\left[\mathrm{Rh}(\mathrm{CO})_{2} \mathrm{Cl}\right]_{2}(6 \mathrm{mg}, 0.015 \mathrm{mmol})$ added at $70{ }^{\circ} \mathrm{C}$. Stirring was continued at $90^{\circ} \mathrm{C}$ for $2 \mathrm{~h}$ then another portion of $\left[\mathrm{Rh}(\mathrm{CO})_{2} \mathrm{Cl}\right](6 \mathrm{mg}, 0.015 \mathrm{mmol})$ was added. The total reaction time was $4 \mathrm{~h}$. Upon completion the reaction mixture was cooled to rt, and filtered through a pad of silica gel to afford the title compound $(80 \mathrm{mg}, 66 \%)$. Examination of the ${ }^{1} \mathrm{H}$ 
NMR indicated presence of two isomers in a ratio of $2.5: 1$. The isomers were separated by semi-preparative HPLC (hexanes-EtOAc, $24: 1$, v/v).

(Major isomer $\left.-\mathrm{R}_{\mathrm{t}}=35 \mathrm{~min}\right){ }^{1} \mathrm{H} \mathrm{NMR}\left(\mathrm{CDCl}_{3}, 300 \mathrm{MHz}\right) \delta$ : 7.55-7.51 (m, 2H), 7.42-7.34 (m, 3H), 7.26-7.16 (m, 5H), 5.44 (q, $J=7.0 \mathrm{~Hz}, 1 \mathrm{H}), 5.13(\mathrm{dd}, J=7.7,2.5 \mathrm{~Hz}, 1 \mathrm{H}), 4.98$ $(\mathrm{dt}, J=7.7,5.1 \mathrm{~Hz}, 1 \mathrm{H}), 4.19(1 / 2 \mathrm{AB}, J=13.7 \mathrm{~Hz}, 1 \mathrm{H}), 3.75(\mathrm{~s}, 3 \mathrm{H}), 3.36(1 / 2 \mathrm{AB}, J=$ $16.6 \mathrm{~Hz}, 1 \mathrm{H}), 3.14(1 / 2 \mathrm{AB}, J=13.7 \mathrm{~Hz}, 1 \mathrm{H}), 2.84(1 / 2 \mathrm{AB}, J=14.9 \mathrm{~Hz}, 1 \mathrm{H}), 2.71-2.58$ (m, 2H), $1.62(\mathrm{~d}, J=7.0 \mathrm{~Hz}, 3 \mathrm{H}) ;{ }^{13} \mathrm{C} \mathrm{NMR}\left(\mathrm{CDCl}_{3}, 75 \mathrm{MHz}\right) \delta: 172.7,171.2,136.9$, $136.4,130.9,130.4,129.9,129.6,128.2,128.1,128.0,126.8,122.2,120.0,68.3,52.4$, 40.3, 35.6, 33.1, 13.4; IR (thin film) v 2949, 1736, 1663, 1635, 1446, $1352 \mathrm{~cm}^{-1}$; MS m/z (\%) 375 (38), 344 (8), 316 (15), 284 (22), 105 (100); EI-HRMS Calcd for $\mathrm{C}_{24} \mathrm{H}_{25} \mathrm{NO}_{3}$ $\left[\mathrm{M}^{+}\right], \mathrm{m} / \mathrm{z}$ 375.1834; found 375.1831.

(Minor isomer $\left.-\mathrm{R}_{\mathrm{t}}=37 \mathrm{~min}\right){ }^{1} \mathrm{H} \mathrm{NMR}\left(\mathrm{CDCl}_{3}, 300 \mathrm{MHz}\right) \delta$ : 7.54-7.51 (m, 2H), 7.45-7.34 (m, 3H), 7.25-7.17 (m, 5H), 5.38 (br q, 1H), 5.16 (dd, $J=7.6,2.6 \mathrm{~Hz}, 1 \mathrm{H}), 5.04$ (dt, $J=$ 7.8, $4.8 \mathrm{~Hz}, 1 \mathrm{H}), 4.18(1 / 2 \mathrm{AB}, J=13.7 \mathrm{~Hz} .1 \mathrm{H}), 3.71(\mathrm{~s}, 3 \mathrm{H}), 3.16-3.11(\mathrm{~m}, 1 \mathrm{H}), 3.08$ $(1 / 2 \mathrm{AB}, J=13.7 \mathrm{~Hz}, 1 \mathrm{H}), 2.90(1 / 2 \mathrm{AB}, J=14.3 \mathrm{~Hz}, 1 \mathrm{H}), 2.75(\mathrm{dd}, J=17.6,8.2 \mathrm{~Hz}$, $1 \mathrm{H}), 2.36(1 / 2 \mathrm{AB}, J=14.3 \mathrm{~Hz}, 1 \mathrm{H}), 1.58(\mathrm{~d}, J=6.9 \mathrm{~Hz}, 3 \mathrm{H}) ;{ }^{13} \mathrm{C} \mathrm{NMR}\left(\mathrm{CDCl}_{3}\right.$, $75 \mathrm{MHz}) \delta: 172.8,171.1,137.0,136.5,130.9,130.6,130.4,128.1,126.7,121.8,120.0$, $69.2,52.0,44.2,40.0,26.3,13.1$; IR (thin film) v 2948, 1736, 1664, 1635, 1446, 1353 $\mathrm{cm}^{-1}$; MS m/z (\%) 375 (45), 344 (26), 316 (47), 284 (22), 105 (100); EI-HRMS Calcd for $\mathrm{C}_{24} \mathrm{H}_{25} \mathrm{NO}_{3}\left[\mathrm{M}^{+}\right], \mathrm{m} / \mathrm{z}$ 375.1834; found 375.1850 . 

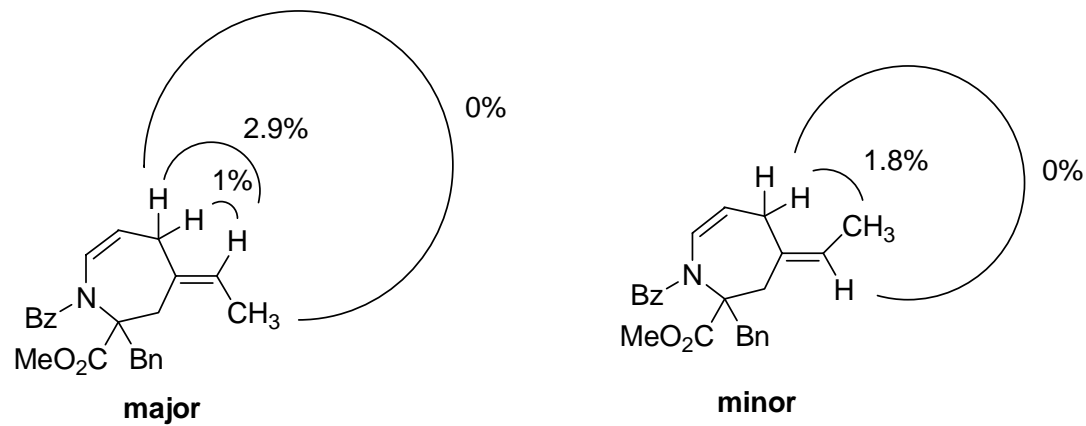

nOe signals of the two isomers of 8 (Table 1, entry 12)
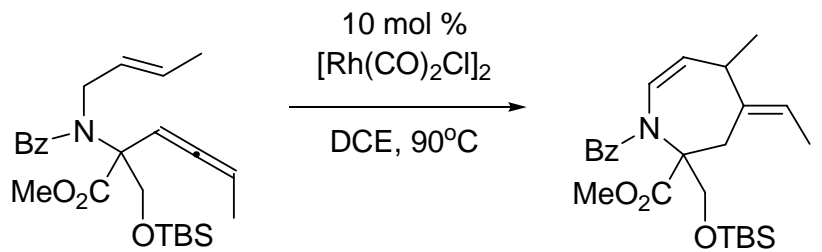

8 (Table 1, entry 13)

\section{1-Benzoyl-2-(tert-butyl-dimethylsilyloxymethyl)-4-ethylidene-5-methyl-2,3,4,5-}

tetrahydro-1H-azepine-2-carboxylic acid methyl ester (8, Table 1, entry 13). Followed general procedure for the rhodium catalyzed azepine formation by cycloisomerization of ene-allenes (page S22):

2-(Benzoylbut-2-enylamino)-2-(tert-butyldimethylsilyloxymethyl)-hexa-3,4-dienoic acid methyl ester (32 mg, $0.072 \mathrm{mmol}),\left[\mathrm{Rh}(\mathrm{CO})_{2} \mathrm{Cl}\right]_{2}$ in two portions of $(1.5 \mathrm{mg}, 0.0036$ mmol). First portion of $\left[\mathrm{Rh}(\mathrm{CO})_{2} \mathrm{Cl}\right]_{2}$ added at $\mathrm{rt}$. The total reaction time was $4 \mathrm{~h}$.

Yield (23 mg, $72 \%)$ as a $4.5: 1$ mixture of diastereomers distinguishable by ${ }^{1} \mathrm{H}$ NMR. ${ }^{1} \mathrm{H}$ NMR $\left(\mathrm{CDCl}_{3}, 300 \mathrm{MHz}\right) \delta: 7.57-7.52(\mathrm{~m}, 2 \mathrm{H}), 7.42-7.33(\mathrm{~m}, 3 \mathrm{H}), 5.93(\mathrm{~d}, J=9.0 \mathrm{~Hz}$, $1 \mathrm{H})^{* *}, 5.89(\mathrm{dd}, J=8.3,2.6 \mathrm{~Hz}, 1 \mathrm{H})^{*}, 5.55(\mathrm{q}, J=6.8 \mathrm{~Hz}, 1 \mathrm{H}), 4.97(\mathrm{dd}, J=8.7,6.8 \mathrm{~Hz}$, $1 \mathrm{H})^{* *}, 4.83(\mathrm{dd}, J=8.3,3.9 \mathrm{~Hz}, 1 \mathrm{H})^{*}, 4.66(\mathrm{~d}, J=9.9 \mathrm{~Hz}, 1 \mathrm{H})^{*}, 4.59(\mathrm{~d}, J=10.0 \mathrm{~Hz}$, $1 \mathrm{H})^{* *}, 3.94(\mathrm{~d}, J=9.9 \mathrm{~Hz}, 1 \mathrm{H})^{* *}, 3.89(\mathrm{~d}, J=9.9 \mathrm{~Hz}, 1 \mathrm{H})^{*}, 3.69(\mathrm{~s}, 3 \mathrm{H})^{* *}, 3.69(\mathrm{~s}$, $3 \mathrm{H})^{*}, 3.46(\mathrm{br} \mathrm{s}, 1 \mathrm{H}), 3.04(\mathrm{~d}, J=14.9 \mathrm{~Hz}, 1 \mathrm{H}), 2.74(\mathrm{~d}, J=14.6 \mathrm{~Hz}, 1 \mathrm{H})^{* *}, 2.64(\mathrm{~d}, J=$ 
$14.8 \mathrm{~Hz}, 1 \mathrm{H})^{*}, 1.58(\mathrm{~d}, J=6.8 \mathrm{~Hz}, 3 \mathrm{H}), 1.33(\mathrm{~d}, J=7.0 \mathrm{~Hz}, 3 \mathrm{H})^{* *}, 1.19(\mathrm{~d}, J=7.2 \mathrm{~Hz}$, $3 \mathrm{H})^{*}, 0.86(\mathrm{~s}, 9 \mathrm{H}), 0.07(\mathrm{~s}, 3 \mathrm{H})^{*}, 0.06(\mathrm{~s}, 3 \mathrm{H})^{* *}, 0.03(\mathrm{~s}, 3 \mathrm{H})^{* *}, 0.02(\mathrm{~s}, 3 \mathrm{H}) * * ;{ }^{13} \mathrm{C}$ NMR $\left(\mathrm{CDCl}_{3}, 75 \mathrm{MHz}\right) \delta: 171.5,171.4,136.2,135.3,130.4,129.2,128.6,128.0,124.1$, $121.9,69.2,64.6,52.2,37.8,32.4,25.8,21.9,18.0,13.6,-5.3,-5.6$; IR (thin film) v 2953, 1734, 1641, $1342 \mathrm{~cm}^{-1}$; MS m/z (\%) 443 (67), 428 (49), 413 (37), 386 (40), 105 (100); EI-HRMS Calcd for $\mathrm{C}_{25} \mathrm{H}_{37} \mathrm{NO}_{4} \mathrm{Si}\left[\mathrm{M}^{+}\right], m / z$ 443.2492; found 443.2500.

* denotes the major diastereomer; $* *$ denotes the minor diastereomer.

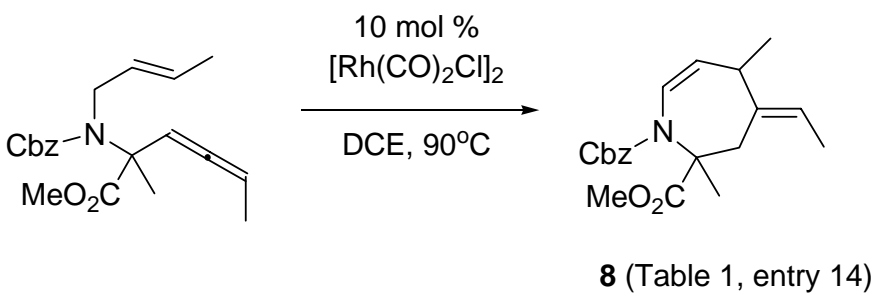

\section{4-Ethylidene-2,5-dimethyl-2,3,4,5-tetrahydroazepine-1,2-dicarboxylic acid 1-benzyl}

ester 2-methyl ester (8, Table 1, entry 14). Followed general procedure for the rhodium catalyzed azepine formation by cycloisomerization of ene-allenes (page S22):

2-(Benzyloxycarbonyl-but-2-enyl-amino)-2-methyl-hexa-3,4-dienoic acid methyl ester (105 mg, $0.306 \mathrm{mmol}),\left[\mathrm{Rh}(\mathrm{CO})_{2} \mathrm{Cl}\right]_{2}$ in two portions $(2 \times 6 \mathrm{mg}, 0.015 \mathrm{mmol})$. First portion of $\left[\mathrm{Rh}(\mathrm{CO})_{2} \mathrm{Cl}\right]_{2}$ was added at $70{ }^{\circ} \mathrm{C}$ under Ar. The total reaction time was $4 \mathrm{~h}$. The crude mixture was purified by flash chromatography (hexanes-EtOAc, $30: 1$ to 20 : $1, \mathrm{v} / \mathrm{v})$ to afford the title compound ( $70 \mathrm{mg}, 63 \%)$. Best yield is obtained when minimal amount of silica gel is used for purification as the compound appeared to decompose upon exposure to silica gel.

${ }^{1} \mathrm{H}$ NMR $\left(300 \mathrm{MHz}, \mathrm{CDCl}_{3}\right)$, $8: 7.36-7.29(\mathrm{~m}, 5 \mathrm{H}), 6.24(\mathrm{~d}, J=8.8 \mathrm{~Hz}, 1 \mathrm{H}), 5.48(\mathrm{q}, J=$ $6.9 \mathrm{~Hz}, 1 \mathrm{H}), 5.19(1 / 2 \mathrm{AB}, J=12.3 \mathrm{~Hz}, 1 \mathrm{H}), 5.10(1 / 2 \mathrm{AB}, J=12.3 \mathrm{~Hz}, 1 \mathrm{H}), 4.91(\mathrm{dd}, J$ $=8.8,4.1 \mathrm{~Hz}, 1 \mathrm{H}), 3.66(\mathrm{br} \mathrm{s}, 3 \mathrm{H}), 3.23(\mathrm{~m}, 1 \mathrm{H}), 2.73(1 / 2 \mathrm{AB}, J=14.6 \mathrm{~Hz}, 1 \mathrm{H}), 2.51$ 
$(1 / 2 \mathrm{AB}, J=14.6 \mathrm{~Hz}, 1 \mathrm{H}), 1.61(\mathrm{~s}, 3 \mathrm{H}), 1.56(\mathrm{~d}, J=6.9 \mathrm{~Hz}, 3 \mathrm{H}), 1.14(\mathrm{~d}, J=7.2 \mathrm{~Hz}$, $1 \mathrm{H}) ;{ }^{13} \mathrm{C} \mathrm{NMR}\left(\mathrm{CDCl}_{3}, 75 \mathrm{MHz}\right) \delta: 173.6,154.8,135.5,128.6,128.2,123.9,123.0$, $122.1,67.8,65.9,52.5,38.2,37.3,23.7,21.6,14.0$; IR (thin film) 3400, 2951, 1742, $1710,1456,1387,1304 \mathrm{~cm}^{-1}$. MS (GC/MS) m/z $284\left[\mathrm{M}-59^{+}\right]$.

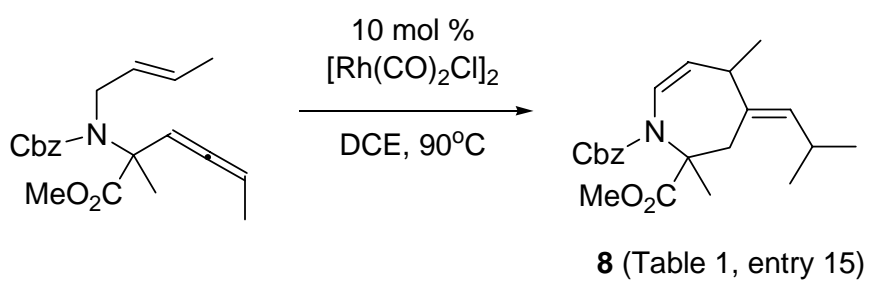

4-Isobutylidene-2,5-dimethyl-2,3,4,5-tetrahydroazepine-1,2-dicarboxylic acid 1benzyl ester 2-methyl ester (8, Table 1, entry 15$)$. Followed general procedure for the rhodium catalyzed azepine formation by cycloisomerization of ene-allenes (page S22): 2-(Benzyloxycarbonylbut-2-enylamino)-2,6-dimethylhepta-3,4-dienoic acid methyl ester (35 mg, $0.094 \mathrm{mmol}),\left[\mathrm{Rh}(\mathrm{CO})_{2} \mathrm{Cl}\right]_{2}(3.5 \mathrm{mg}, 0.0094 \mathrm{mmol})$ added in a single portion at rt. Heated at $90{ }^{\circ} \mathrm{C}$ for $5 \mathrm{~h}$.

Yield (18 mg, $51 \%$ ). Rapid decomposition was observed after purification.

${ }^{1} \mathrm{H} \mathrm{NMR}\left(\mathrm{CDCl}_{3}, 300 \mathrm{MHz}\right) \delta: 7.35$ (s, 5H), $6.23(\mathrm{~d}, J=7.2 \mathrm{~Hz}, 1 \mathrm{H}), 5.22-5.09$ (m, 3H), 4.99 (s, 1H), 3.67 (br s, 3H), 3.29 (s, 1H), $2.72(1 / 2 \mathrm{AB}, J=14.9 \mathrm{~Hz}, 1 \mathrm{H}), 2.53(1 / 2 \mathrm{AB}$, $J=15.0 \mathrm{~Hz}, 1 \mathrm{H}), 2.48-2.30(\mathrm{~m}, 1 \mathrm{H}), 1.61(\mathrm{~s}, 3 \mathrm{H}), 1.15(\mathrm{~d}, J=7.0 \mathrm{~Hz}, 3 \mathrm{H}), 0.91(\mathrm{~d}, J=$ $6.4 \mathrm{~Hz}, 6 \mathrm{H}) ;{ }^{13} \mathrm{C} \mathrm{NMR}\left(\mathrm{CDCl}_{3}, 75 \mathrm{MHz}\right) \delta: 173.5,154.7,135.0,131.9,128.6,128.2$, $124.2,67.7,65.2,52.3,38.2,36.3,29.8,27.4,23.4,20.4$; IR (thin film) v 2956, 1743, $1710,1306 \mathrm{~cm}^{-1}$.
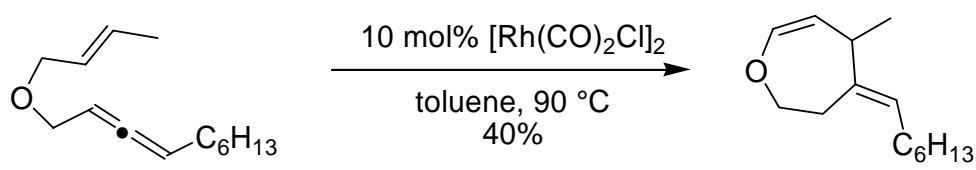

10 (Table 1, entry 16) 
4-(E)-Heptylidene-5-methyl-2,3,4,5-tetrahydrooxepine (10, Table 1, entry 16): 1-[(E)-2-Butenyloxy]-5,5-dimethyl-2,3-hexadiene (9, Table 1, entry 16, $20 \mathrm{mg}, 0.096$ mmol) and toluene- $\mathrm{d}_{8}(0.5 \mathrm{~mL})$ were placed in a dry NMR tube. The tube was flushed with $\mathrm{N}_{2}$ for 1 min. After a ${ }^{1} \mathrm{H}$ NMR spectrum was taken, 1,2-dichloroethane $(1 \mu \mathrm{L}$, internal standard $)$ and $\left[\mathrm{Rh}(\mathrm{CO})_{2} \mathrm{Cl}\right]_{2}(3.7 \mathrm{mg}, 0.0095 \mathrm{mmol})$ were added and the NMR tube was sealed. The NMR tube was then heated at $90{ }^{\circ} \mathrm{C}$ for 90 min at which time ${ }^{1} \mathrm{H}$ NMR indicated total consumption of the starting material. The yield of the product was determined by the comparison of the integrations of the olefinic protons of the product to that of the internal standard as to be 53\%. The product was then removed from the NMR tube and purified by flash chromatography $\left(\mathrm{SiO}_{2}\right.$, pentane $:$ ether $\left.=50: 1\right)$ to afford the product as a colorless oil $(7.9 \mathrm{mg}, 40 \%): \quad{ }^{1} \mathrm{H}$ NMR $\left(300 \mathrm{MHz}, \mathrm{CDCl}_{3}\right) \delta 6.10(\mathrm{dd}, J=$ 1.6, $7.3 \mathrm{~Hz}, 1 \mathrm{H}), 5.22(\mathrm{t}, J=7.0 \mathrm{~Hz}, 1 \mathrm{H}), 4.45(\mathrm{dd}, J=4.9,7.3 \mathrm{~Hz}, 1 \mathrm{H}), 4.16-4.03(\mathrm{~m}$, $2 \mathrm{H}), 3.16(\mathrm{dq}, J=7.0,7.0 \mathrm{~Hz}, 1 \mathrm{H}), 2.70-2.53(\mathrm{~m}, 2 \mathrm{H}), 2.98(\mathrm{dt}, J=6.8,7.0 \mathrm{~Hz}, 2 \mathrm{H})$, 1.43-1.21 (m, 8H), $1.17(\mathrm{~d}, J=7.0 \mathrm{~Hz}, 3 \mathrm{H}), 0.89$ (t, $J=6.4 \mathrm{~Hz}, 3 \mathrm{H}) ;{ }^{13} \mathrm{C} \mathrm{NMR}(75 \mathrm{MHz}$, $\left.\mathrm{CDCl}_{3}\right) \delta 144.3,140.3,123.7,111.0,69.7,39.1,31.8,31.2,29.7,29.0,27.5,22.6,21.5$, 14.1; IR (neat) $\vee 1650 \mathrm{~cm}^{-1}$; EI-HRMS calcd for $\left[\mathrm{M}^{+}\right] \mathrm{m} / \mathrm{z} 208.1827$, found 208.1827.
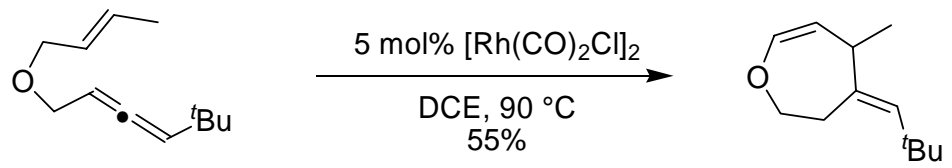

10 (Table 1, entry 17)

\section{4-[2,2-Dimethyl-(E)-propylidene]-5-methyl-2,3,4,5-tetrahydrooxepine}

$(\mathbf{1 0}$,

Table 1, entry 17): This compound was prepared following the procedure for the preparation of 6 (Table 1, entry 1) from 1-[(E)-2-butenyloxy]-5,5-dimethyl-2,3-hexadiene (9, Table 1, entry 17, $20.4 \mathrm{mg}, 0.11 \mathrm{mmol})$ and $\left[\mathrm{Rh}(\mathrm{CO})_{2} \mathrm{Cl}\right]_{2}(2.2 \mathrm{mg}, 0.0055 \mathrm{mmol})$ in 
1,2-dichloroethane $(0.5 \mathrm{~mL})$ in $\mathrm{Ar}$ atmosphere (1 atm), after flash chromatography $\left(\mathrm{SiO}_{2}\right.$, hexanes : ether $=50: 1)$ as a colorless oil $(11.3 \mathrm{mg}, 55 \%):{ }^{1} \mathrm{H}$ NMR $\left(300 \mathrm{MHz}, \mathrm{CDCl}_{3}\right)$ $\delta 6.11(\mathrm{dd}, J=1.2,7.4 \mathrm{~Hz}, 1 \mathrm{H}), 5.25(\mathrm{~s}, 1 \mathrm{H}), 4.48(\mathrm{dd}, J=5.4,7.3 \mathrm{~Hz}, 1 \mathrm{H}), 4.10(\mathrm{ddd}, J$ $=3.2,7.0,11.7 \mathrm{~Hz}, 1 \mathrm{H}), 4.00(\mathrm{ddd}, J=3.2,7.0,11.7 \mathrm{~Hz}, 1 \mathrm{H}), 3.03(\mathrm{dq}, J=7.0,7.0 \mathrm{~Hz}$, 1H), $2.81(\mathrm{ddd}, J=2.3,7.8,15.7 \mathrm{~Hz}), 2.68(\mathrm{ddd}, J=2.3,7.8,15.7 \mathrm{~Hz}, 1 \mathrm{H}), 1.15(\mathrm{~d}, J=$ $7.1 \mathrm{~Hz}, 3 \mathrm{H}), 1.11(\mathrm{~s}, 9 \mathrm{H}) ;{ }^{13} \mathrm{C} \mathrm{NMR}\left(75 \mathrm{MHz}, \mathrm{CDCl}_{3}\right) \delta 144.2,139.8,134.0,111.3,70.2$, 41.2, 31.9, 31.4, 30.2, 22.1; IR (neat) $v 1650 \mathrm{~cm}^{-1}$; EI-HRMS calcd for $\mathrm{C}_{12} \mathrm{H}_{20} \mathrm{O}\left[\mathrm{M}^{+}\right] \mathrm{m} / \mathrm{z}$ 180.1513, found 180.1514 .
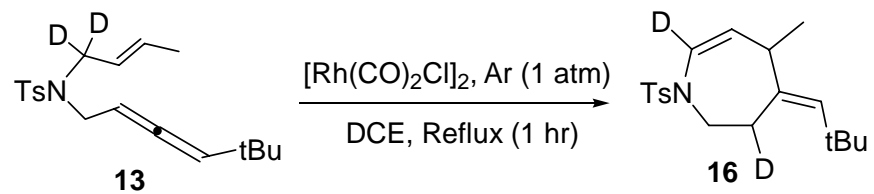

\section{4-(E)-(2,2-Dimethyl-propylidene)-5-methyl-1-(4-methyl-benzenesulfonyl)-2,3,4,5-}

tetrahydro-1H-azepine-3,7- $\boldsymbol{d}_{2}(\mathbf{1 6})$. The procedure for preparation of $6 \mathbf{i}$ was followed using ene-allene $13(28 \mathrm{mg}, 0.083 \mathrm{mmol}),\left[\mathrm{Rh}(\mathrm{CO})_{2} \mathrm{Cl}\right]_{2}(3.2 \mathrm{mg}, 0.0082 \mathrm{mmol})$, and dichloroethane $(500 \mu \mathrm{L})$. A color change was apparent within the first 15 min of reaction time. Reaction progress was monitored by gas chromatography, reaching completion after $1 \mathrm{~h}$. The reaction, upon purification by silica gel chromatography (ethyl acetatehexanes 1:19), afforded $25 \mathrm{mg}$ of colorless oil 16 in 89\% yield. ${ }^{1} \mathrm{H}$ NMR (300 MHz, $\left.\mathrm{C}_{6} \mathrm{D}_{6}\right) \delta: 7.69(1 / 2 \mathrm{AB} \mathrm{q}, J=8.3 \mathrm{~Hz}, 2 \mathrm{H}), 6.74(1 / 2 \mathrm{AB} \mathrm{q}, J=8.0 \mathrm{~Hz}, 2 \mathrm{H}), 5.21$ (dd, $J=$ $1.0,1.0 \mathrm{~Hz}, 1 \mathrm{H}), 4.60(\mathrm{~d}, J=5.4 \mathrm{~Hz}, 1 \mathrm{H}), 3.56(\mathrm{dd}, J=13.5,7.8 \mathrm{~Hz}, 1 \mathrm{H}), 3.38(\mathrm{dd}, J=$ 13.5, 3.5 Hz, 1H), 2.82-2.71 (m, 1H), 2.49-2.43 (m, 1H), 1.85 (s, 3H), 0.97 (s, 9H), 0.91 $(\mathrm{d}, J=7.2,3 \mathrm{H}) ;{ }^{13} \mathrm{C} \mathrm{NMR}\left(75 \mathrm{MHz}, \mathrm{CDCl}_{3}\right) \delta: 143.8,138.1,136.8,135.6,130.0,127.2$, 125.7 (t), 119.1, 48.1, 41.2, 31.7, 31.6, 29.3 (t), 21.8, 21.4; IR (neat) 2958, 2869, 1711, 
1624, 1460, $1353 \mathrm{~cm}^{-1}$; MS m/z (\%) 335 (45), 320 (100), 180 (52), 124 (55), 91 (82); EIHRMS calcd for $\mathrm{C}_{19} \mathrm{H}_{25} \mathrm{D}_{2} \mathrm{NO}_{2} \mathrm{~S}$ : 335.1888; found: 335.1889 . 